\title{
Counting strings, wound and bound
}

\author{
Sujay K. Ashok, ${ }^{a}$ Suresh Nampuri ${ }^{b}$ and Jan Troost $^{b}$ \\ ${ }^{a}$ Institute of Mathematical Sciences, \\ C.I.T Campus, Taramani, Chennai, 600113 India \\ ${ }^{b}$ Laboratoire de Physique Théorique, ${ }^{1}$ Ecole Normale Supérieure, \\ 24 rue Lhomond, F-75231 Paris Cedex 05, France \\ E-mail: sashok@imsc.res.in, nampuri@gmail.com, troost@lpt.ens.fr
}

ABSTRACT: We analyze zero mode counting problems for Dirac operators that find their origin in string theory backgrounds. A first class of quantum mechanical models for which we compute the number of ground states arises from a string winding an isometric direction in a geometry, taking into account its energy due to tension. Alternatively, the models arise from deforming marginal bound states of a string winding a circle, and moving in an orthogonal geometry. After deformation, the number of bound states is again counted by the zero modes of a Dirac operator. We count these bound states in even dimensional asymptotically linear dilaton backgrounds as well as in Euclidean Taub-NUT. We show multiple pole behavior in the fugacities keeping track of a U(1) charge. We also discuss a second class of counting problems that arises when these backgrounds are deformed via the application of a heterotic duality transformation. We discuss applications of our results to Appell-Lerch sums and the counting of domain wall bound states.

KEYwords: Conformal Field Models in String Theory, Superstring Vacua, Extended Supersymmetry

ARXIV EPRINT: 1302.1045

\footnotetext{
${ }^{1}$ Unité Mixte du CNRS et de l'Ecole Normale Supérieure associée à l'université Pierre et Marie Curie 6, UMR 8549.
} 


\section{Contents}

1 Introduction $\quad 2$

2 Indices, quantum mechanics and field theory 3

2.1 The supersymmetric quantum mechanics: manifolds with isometry 3

2.2 Twisted dimensional reduction 4

2.3 A marginal bound state problem 5

3 Asymptotically linear dilaton spaces $\quad 6$

3.1 The two-dimensional cigar $\quad 6$

3.2 The four-dimensional asymptotically linear dilaton background 8

$\begin{array}{llr}3.2 .1 & \text { Linear equations } & 9\end{array}$

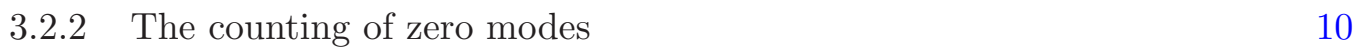

3.3 The six-dimensional asymptotically linear dilaton background 11

$\begin{array}{ll}3.4 & \text { The physics underlying the index formulas } \\ \end{array}$

4 Duality rotated asymptotically linear dilaton spaces $\quad \mathbf{1 6}$

$\begin{array}{ll}4.1 \text { Duality rotated backgrounds } & 16\end{array}$

$\begin{array}{lll}\text { 4.1.1 Asymptotically linear dilaton dyons } & 17\end{array}$

$\begin{array}{lll}4.2 & \text { Charged Dirac indices } & 18\end{array}$

$\begin{array}{lll}\text { 4.2.1 Four-dimensional asymptotically linear dilaton dyon } & 19\end{array}$

5 Conclusions and future directions $\quad 20$

5.1 Mock modular forms 21

5.2 Counting domain wall bound states 23

A First order differential equations $\quad \mathbf{2 4}$

B Fibrations over $\mathbb{C P}^{N-1} \quad \mathbf{2 5}$

B.1 The sphere as a circle fibration $\quad 25$

B.2 Asymptotically linear dilaton fibrations 27

$\begin{array}{lll}\text { B.2.1 Asymptotic linear dilaton fibration over } \mathbb{C P}^{1} & 27\end{array}$

$\begin{array}{lll}\text { B.2.2 Asymptotic linear dilaton fibration over } \mathbb{C P}^{2} & 28\end{array}$

C Euclidean Taub-NUT backgrounds $\quad 28$

C.1 Euclidean Taub-NUT with self-dual gauge field 29

C.2 The heterotic Euclidean Taub-NUT dyon 30

$\begin{array}{ll}\text { C.2.1 The background } & 30\end{array}$

C.2.2 Dirac index for a charged spinor on the Taub-NUT dyon 31

$\begin{array}{ll}\text { D Conventions for spinors } & 31\end{array}$ 


\section{Introduction}

It is often useful to reduce a physical problem in a higher dimensional theory to a property of a lower dimensional model that is more easily solved. In this paper, we concentrate on the calculation of Dirac indices in a class of supersymmetric quantum mechanics models. These supersymmetric quantum mechanics problems arise when we study the motion of a string on a target manifold with an isometry. When we wind the string in the isometric direction, we perform a reduction from $1+1$ dimensions to 1 dimension, and we obtain a supersymmetric quantum mechanics with potential [1]. The ground states correspond to bound states of wound strings.

Another context in which the same model arises is when we study the non-trivial space with isometry, tensored with a circle. When we now wind the string on the circle, it is often marginally bound to the non-trivial space, while preserving supersymmetry. When reducing our attention to its center of mass motion on the geometry, it gives rise to a supersymmetric quantum mechanics. It is then interesting to find a natural deformation of the resulting supersymmetric quantum mechanics such that the marginal bound state spectrum is deformed to a spectrum of bound states. We can then proceed to count the latter. The natural deformation consists in introducing a small winding along the isometric direction, as above.

We employ also a second deformation technique in which we deform the background itself. We imagine embedding our solution in some heterotic or Kaluza-Klein reduced supergravity theory and implement a small duality transformation on the isometric direction and some gauge direction in the supergravity theory, to introduce a non-trivial gauge field in the supersymmetric quantum mechanics. We then consider charged excitations, coupled to the gauge field. Both techniques that we describe enter in the realm of the general technique of adding potentials to moduli spaces.

We apply our two deformation techniques to a class of string backgrounds which are non-compact and which have an asymptotically linear dilaton. They were first written down in [2] and were later studied in the context of gauged linear sigma models [3, 4]. We will refer to these as asymptotically linear dilaton spaces. The simplest example of such a space is the well studied supersymmetric cigar conformal field theory in two dimensions. Index theorems for these non-compact backgrounds need to take into account subtle boundary contributions. We find it convenient to proceed by an explicit counting of states, and as a bonus provide a construction of the bound state solutions in these backgrounds.

We also apply the methods we develop to the Euclidean Taub-NUT space in four dimensions, and make a technical improvement to the existing method [5] of counting bound states. The latter index has already found applications to the counting of monopole bound states [6] and to the counting of one quarter BPS dyons in $\mathcal{N}=4$ four-dimensional compactifications of string theory [7]. Likewise, we will highlight potential applications of our results on asymptotically linear dilaton backgrounds in the realm of domain wall bound states in gauge theories.

Another application of the index formulas is a re-derivation of the mock modular form that arises in the calculation of the elliptic genus of the cigar conformal field theory. This 
is done by first defining a generating function that keeps track of the Dirac zero modes in a given momentum and winding sector. This generating function is then lifted to the elliptic genus by assigning appropriate fugacities that keep track of the momentum and winding quantum numbers, and by adding oscillator contributions. The index calculation gives a direct physical understanding of the double sum form of Appell-Lerch sums featuring in physical counting problems.

Our paper is structured as follows. We start in section 2 by reviewing supersymmetric quantum mechanics with a potential on target manifolds with an isometry and set up the wound bound state counting problem. In section 3 we introduce the asymptotically linear dilaton spaces of our interest and introduce all the technology needed to compute the Dirac indices that depend on a winding number and a momentum charge. In section 4 we introduce the heterotic backgrounds that we generate by duality transformations, and the details on how to compute the indices in these backgrounds. In the final section 5 we conclude and indicate possible applications of these counting formulas. Some technical details are provided in appendices $\mathrm{A}$ and $\mathrm{B}$. In appendix $\mathrm{C}$ we illustrate how our techniques simplify the calculation of the index on Euclidean Taub-NUT with self-dual gauge field.

\section{Indices, quantum mechanics and field theory}

In this section, we review a class of supersymmetric quantum mechanics models which are one-dimensional non-linear sigma-models. We recall the existence of a potential that is consistent with supersymmetry when the target manifold of the model has an isometry, and how that potential arises naturally from a twisted dimensional reduction from $1+1$ dimensions [1]. The model then corresponds to the center of mass motion of a wound string, and the potential arises from the energy of the tensionful string. Secondly, we mention how a marginally bound string, wound transversely to such a target space geometry after a small deformation also gives rise to the supersymmetric quantum mechanics with Killing vector potential.

\subsection{The supersymmetric quantum mechanics: manifolds with isometry}

We consider a $0+1$ dimensional sigma-model with at least one supersymmetry, namely a supersymmetric quantum mechanics. The target manifold $M$ with metric $G$ permits one Killing isometry generated by the vector field $K$. We have therefore the Killing equation:

$$
\mathcal{L}_{K} G=0
$$

The Lagrangian of the model includes a kinetic term and a potential term associated to the existence of the Killing isometry [1]:

$$
L=\frac{1}{2}\left(G_{\mu \nu} \dot{X}^{\mu} \dot{X}^{\nu}+i G_{\mu \nu} \psi^{\mu} \mathcal{D}_{\tau} \psi^{\nu}-G_{\mu \nu} K^{\mu} K^{\nu}-i D_{\mu} K_{\nu} \psi^{\mu} \psi^{\nu}\right)
$$

The dot represents a derivative with respect to the world line time $\tau$, while the derivative $\mathcal{D}_{\tau}$ is the covariant derivative of the tangent space valued world line fermion. If we define 
the vielbein $e_{\mu}^{a}$, and the world line fermion $\psi^{a}=\psi^{\mu} e_{\mu}^{a}$, then after quantization we find the canonical commutators:

$$
\begin{aligned}
{\left[X^{\mu}, p_{\nu}\right] } & =i \delta_{\nu}^{\mu} \\
\left\{\psi^{a}, \psi^{b}\right\} & =\delta^{a b} .
\end{aligned}
$$

The fermionic modes form a Clifford algebra. After quantization, they give rise to a Hilbert space corresponding to a space-time fermion. The super-covariant momentum:

$$
\pi_{\mu}=p_{\mu}-\frac{i}{4} \omega_{\mu a b}\left[\psi^{a}, \psi^{b}\right]
$$

acts like a covariant derivative on space-time spinors: $\pi_{\mu}=-i D_{\mu}$. The supercharge $Q$ is given by the formula:

$$
Q=\psi^{\mu}\left(-i D_{\mu}-K_{\mu}\right)
$$

while the central charge $Z$ is:

$$
Z=K^{\mu} \pi_{\mu}-\frac{i}{2}\left(D_{\mu} K_{\nu}\right) \psi^{\mu} \psi^{\nu}
$$

It is proportional to the Lie derivative acting on spinors. The supersymmetric quantum mechanics allows for a $\mathbb{Z}_{2}$ grading (world line fermion number) given by the operator $\prod_{a} \psi^{a}$ which anti-commutes with the Dirac operator:

$$
\not D=\sqrt{2} Q=\gamma^{\mu}\left(-i D_{\mu}-K_{\mu}\right) .
$$

In most of our paper, we concentrate on computing the ground state contributions to the Witten index, namely the difference between the number of world line bosonic and fermionic zero modes of the Dirac operator in a given set of models. The index is defined for each fixed central charge sector.

\subsection{Twisted dimensional reduction}

In this section, we recall how the above supersymmetric quantum mechanics model can be obtained from a Scherk-Schwarz reduction [1]. We start with a $(1,0)$ supersymmetric theory in $1+1$ dimensions. We will consider a compact space-like $\sigma$-direction of period $2 \pi$. We take the action to be:

$$
S=\frac{1}{4 \pi} \int d^{2} \sigma\left(G_{\mu \nu}\left(\frac{1}{\alpha^{\prime}}\left(\partial_{\tau} X^{\mu} \partial_{\tau} X^{\nu}-\partial_{\sigma} X^{\mu} \partial_{\sigma} X^{\nu}\right)+i \psi^{\mu} \mathcal{D}_{\tau-\sigma} \psi^{\nu}\right)\right) .
$$

Note that the choice of sign of derivative with respect to $\sigma$ in the fermion kinetic term determines whether we concentrate on the supersymmetric quantum mechanics associated to the $1+1$ dimensional right- or left-movers.

Let us consider the target space isometry generated by the vector field $K$. ScherkSchwarz or twisted dimensional reduction can be carried out by requiring that the dependence of the fields on the $\sigma$ direction is given in terms of the Killing vector that generates the isometry:

$$
\partial_{\sigma} X^{\mu}=K^{\mu} \quad \text { and } \quad \partial_{\sigma} \psi^{\mu}=-\partial_{\nu} K^{\mu} \psi^{\nu} .
$$


We now integrate over the $\sigma$ direction and obtain a quantum mechanical system. Our ansatz ensures that the reduction will be supersymmetric. Plugging this ansatz into the action, we find the dimensionally reduced action:

$$
S=\frac{1}{2} \int d \tau\left(G_{\mu \nu} \frac{1}{\alpha^{\prime}} \partial_{\tau} X^{\mu} \partial_{\tau} X^{\nu}+i G_{\mu \nu} \psi^{\mu} \mathcal{D}_{\tau} \psi^{\nu}-\frac{G_{\mu \nu}}{\alpha^{\prime}} K^{\mu} K^{\nu}+i \psi^{\mu} K_{\mu ; \nu} \psi^{\nu}\right),
$$

which is the action in equation (2.2) provided we put $\alpha^{\prime}=1$, which we will do from now on. The Scherk-Schwarz reduced theory has the interpretation of corresponding to the center of mass motion of a string wound along the isometric direction. This is a natural way in which the supersymmetric quantum mechanics problem that we study arises in string theory.

\subsection{A marginal bound state problem}

As an aside, we want to mention a second way in which the same supersymmetric quantum mechanics problem pops up in string theory. We consider a string living in the space-time $\mathbb{R} \times M \times S^{1} \times X$ and wound on the $S^{1}$ circle. In many instances, a fundamental or Dstring wound on the circle is marginally bound to the geometry $M$. If we suppose that the geometry $M$ admits a $\mathrm{U}(1)$ isometry with compact action, we can associate to it a circle $\tilde{S}^{1}$. It is natural to study the (often truly) bound state counting problem that arises from mixing the $S^{1}$ and $\tilde{S}^{1}$ circles. That introduces a potential on the manifold $M$, arising from the winding energy of the string along the $\tilde{S}^{1}$ circle. If the size of the $\tilde{S}^{1}$ circle shrinks towards the center of the geometry, the string will now be bound to the center of the geometry $M$.

We can put this idea into practice by starting out with a metric on $M \times S^{1}$ :

$$
d s^{2}=G_{M N} d X^{M} d X^{N}=G_{\mu \nu} d X^{\mu} d X^{\nu}+R^{2} d y^{2} .
$$

The coordinate on the $S^{1}$ circle of radius $R$ is $y \equiv y+2 \pi$. By assumption, the metric $G_{\mu \nu}$ on the manifold $M$ admits a Killing isometry $K$. Clearly there is another Killing vector, namely $L=w_{y} \partial_{y}$ where $w_{y}$ represents the winding number of the string around the circle $S^{1}$. We now repeat the Scherk-Schwarz reduction on the worldsheet with the $\sigma$-dependence given by

$$
\partial_{\sigma} X^{M}=\sin \alpha K^{M}+\cos \alpha L^{M} .
$$

After neglecting constant terms, and allowing for no excitations in the decoupled $y$ direction, we again wind up with the world line supercharge:

$$
Q=G_{\mu \nu} \psi^{\mu}\left(-i D_{\mu}-\sin \alpha K^{\mu}\right) .
$$

As before the index counting the number of bosonic minus fermionic ground states is the index of the equivariant Dirac operator $\not_{K}=\not D-i \sin \alpha \not K$. We can alternatively interpret the (dual of the) Killing vector field $A_{\mu}=\sin \alpha G_{\mu \nu} K^{\nu}$ as an abelian gauge field on the manifold $M$.

Our discussion in this section was very general, and can be viewed as one more way to introduce potentials in supersymmetric quantum mechanics, an ubiquitous tool. The technique we introduced above is natural in many string theory contexts. For an example 
application of this tool with Euclidean Taub-NUT as the target space manifold, see [7]. In the following, we evaluate the index for new example geometries $M$ with Killing vector fields $K$. We will compute the index through the explicit solution of the massless Dirac equation on the target manifold $M$.

\section{Asymptotically linear dilaton spaces}

In this section, we introduce the target spaces $M$ on which we concentrate. As string backgrounds, these backgrounds come accompanied by an asymptotically linear dilaton. The manifolds exhibit at least a U(1) isometry group. These supergravity solutions have been found in [2] and discussed further in [3, 4]. Together with an internal conformal field theory, these backgrounds admit microscopic string theory interpretations as near horizon geometries T-dual to configurations of NS5-branes [4]. The two-dimensional example is the cigar geometry [8-10]. We will later show that at least in this example, the index calculation that we perform has an interesting two-dimensional conformal field theory application.

The backgrounds. The metric and dilaton of the supergravity solutions have the form

$$
\begin{aligned}
d s^{2} & =\frac{g_{N}(Y)}{2} d Y^{2}+\frac{2}{N^{2} g_{N}(Y)}\left(d \psi+N A_{\mathrm{FS}}\right)^{2}+2 Y d s_{\mathrm{FS}}^{2} \\
\Phi & =-\frac{N Y}{k} .
\end{aligned}
$$

The function $g_{N}(Y)$ is given by:

$$
g_{N}(Y)=\frac{Y^{N-1}}{N} \frac{e^{\frac{2 N Y}{k}}}{\int_{0}^{Y} t^{N-1} e^{\frac{2 N t}{k}} d t} .
$$

The metric $d s_{\mathrm{FS}}^{2}$ refers to the Fubini-Study metric on the complex projective space $\mathbb{C P}^{N-1}$ and the connection one-form $A_{\mathrm{FS}}$ has differential equal to the Kähler form. The backgrounds we discuss therefore have a $\mathrm{SU}(n) \times \mathrm{U}(1)$ isometry. The $\mathrm{U}(1)$ factor refers to translations along the $\psi$ direction and this is the Killing isometry $K$ we use to twist the Dirac operator. The one-form gauge field $A$ dual to the Killing vector takes the form:

$$
A=\frac{2 w}{N g_{N}(Y)}\left(d \psi+N A_{\mathrm{FS}}\right)
$$

where $w$ is the number of times our string winds the circle parametrized by the coordinate $\psi$ of period $2 \pi N$. In the following, we concentrate on the cases $N=1,2,3$, which correspond to target spaces $M$ of dimension two, four and six. The parameter $k$ is a free parameter in gravity, but is typically quantized in string theory. After T-duality, it can be related to the number of $N S 5$ branes that generate the background $[3,4]$.

\subsection{The two-dimensional cigar}

The first example we concentrate on is the two-dimensional cigar background. This corresponds to a two dimensional conformal field theory with non-trivial metric and dilaton. 
It has a description as a gauged Wess-Zumino-Witten model, which is an exact conformal field theory. The metric and dilaton read:

$$
\begin{aligned}
d s^{2} & =k\left(d \rho^{2}+\tanh ^{2} \rho d \psi^{2}\right) \\
\Phi & =-\log \cosh \rho .
\end{aligned}
$$

It is equivalent to the case $N=1$ in our general discussion by the coordinate transformation $\cosh \rho=e^{\frac{Y}{k}}$. The function $g_{1}(Y)$ is given by

$$
g_{1}(Y)=\frac{2}{k} \frac{e^{\frac{2 Y}{k}}}{e^{\frac{2 Y}{k}}-1} .
$$

The gauge field dual to the angular Killing vector is:

$$
A=\frac{2 w}{g_{1}(Y)} d \psi
$$

The massless Dirac equation. Given these prerequisites, we turn to the solution of the massless Dirac equation for a charged Dirac fermion $\Psi$ on our curved background:

$$
\gamma^{\mu}\left(\partial_{\mu}+\frac{1}{4} \omega_{\mu}^{a b} \gamma_{a b}-i A_{\mu}\right) \Psi=0
$$

In order to solve the Dirac equation we choose the zweibein:

$$
e^{1}=\sqrt{\frac{g_{1}(Y)}{2}} d Y \quad e^{2}=\sqrt{\frac{2}{g_{1}(Y)}} d \psi .
$$

Our conventions for spinors in various dimensions are given in appendix D. We work in the Weyl basis:

$$
\Psi=\left(\begin{array}{l}
\Psi_{-} \\
\Psi_{+}
\end{array}\right)=e^{i n \psi}\left(\begin{array}{l}
G_{1}(Y) \\
G_{2}(Y)
\end{array}\right) .
$$

The solutions to the Dirac equation are then given by

$$
\Psi=e^{i n \psi}\left(\begin{array}{c}
e^{\frac{Y}{k}(n-k w)}\left(g_{1}(Y)\right)^{\frac{1}{4}-\frac{n}{2}} \\
e^{-\frac{Y}{k}(n-k w)}\left(g_{1}(Y)\right)^{\frac{1}{4}+\frac{n}{2}}
\end{array}\right) .
$$

To determine the allowed zero modes, we first study the behavior of the wave functions near the tip and near infinity. Near the tip at $Y=0$, the wave functions take the form

$$
\Psi \sim e^{i n \psi}\left(\begin{array}{l}
Y^{-\frac{1}{4}+\frac{n}{2}} \\
Y^{-\frac{1}{4}-\frac{n}{2}}
\end{array}\right),
$$

while asymptotically, we have the behavior

$$
\Psi \sim e^{i n \psi}\left(\begin{array}{c}
e^{\frac{Y}{k}(n-k w)} \\
e^{-\frac{Y}{k}(n-k w)}
\end{array}\right) .
$$


We need to impose regularity at the tip and require normalizability at infinity. That leads to the following constraints on the variables $n$ and $w$ :

$$
\begin{aligned}
\frac{1}{2} \leq n<k w & \text { for negative chirality modes } \\
k w<n \leq-\frac{1}{2} & \text { for positive chirality modes }
\end{aligned}
$$

Since we have a positive level $k>0$, this implies that the winding $w$ is positive for negative chirality modes while the winding is negative for positive chirality modes. Note that we can think of the asymptotic charge $n-k w$ as an imaginary radial momentum. When $k w$ is half-integer (as is the momentum $n$ ), we can have zero radial momentum. It is clear that if we slightly shift it, we should consider either the positive chirality mode or the negative chirality mode as normalizable. Let's choose a regularization such that for negative momentum $n$, we allow $n=k w$.

These results can be encoded in a partition sum $Z_{2}$ keeping track of the positive and negative chirality spinorial zero-modes, weighted with their momentum $n$ and winding $w$ :

$$
Z_{2}=\left(\sum_{w<0} \sum_{n=[[k w]]}^{-\frac{1}{2}}-\sum_{w>0} \sum_{n=\frac{1}{2}, \frac{3}{2}, \ldots}^{[[k w-\epsilon]]}\right) y_{1}^{n} y_{2}^{w},
$$

where $[[x]]$ is the strict half-integer (i.e. element of $\mathbb{Z}+1 / 2$ ) smaller than or equal to $x$ and $\epsilon$ is a small positive regulator. We have introduced fugacities $y_{1}$ and $y_{2}$ to keep track of the supersymmetric quantum mechanics labeled by the winding $w$, and the conserved charge $n$. The formal sum is convergent for particular values of $y_{1}$ and $y_{2}$. One can rewrite this double summation by first summing over the momentum variable $n$. This leads to the final expression for the generating function of indices:

$$
Z_{2}=\left[\sum_{n<0} \sum_{n-k w \geq 0}-\sum_{n>0} \sum_{n-k w<0}\right] y_{1}^{n} y_{2}^{w} .
$$

Note that our approach to finding wound bound state solutions in the cigar is complementary to the one of [18] where these bound states were uncovered by studying momentum modes in the T-dual geometry. Here we use the Scherk-Schwarz reduction technique to find bound states. Our technique is more generally applicable since it provides the means to compute bound states with both winding and momentum. As we have argued in section 2, it also generalizes to a large class of backgrounds.

\subsection{The four-dimensional asymptotically linear dilaton background}

The next example we tackle is the asymptotically linear dilaton solution in four dimensions:

$$
d s^{2}=\frac{g_{2}(Y)}{2} d Y^{2}+\frac{1}{2 g_{2}(Y)}(d \psi+\cos \theta d \phi)^{2}+\frac{Y}{2}\left(d \theta^{2}+\sin ^{2} \theta d \phi^{2}\right) .
$$

The radial function $g_{2}(Y)$ is given by:

$$
g_{2}(Y)=\frac{8}{k^{2}} \frac{Y}{e^{-\frac{4 Y}{k}}-1+\frac{4 Y}{k}} .
$$


Along with a non-trivial dilaton $\Phi=-\frac{2 Y}{k}$, the background solves the string equations of motion. The central charge of the corresponding conformal field theory can be calculated from the dilaton profile and comes out to be

$$
c=6\left(1+\frac{4}{k}\right) .
$$

The wave functions have a simple dependence on the angular variables $\psi$ and $\phi$. Given the form of the metric in (3.16) the four Dirac components are separable and we choose the ansatz

$$
\Psi=e^{i n \psi+i m \phi}\left(\begin{array}{c}
G_{1}(Y) S_{1}(\theta) \\
\vdots \\
G_{4}(Y) S_{4}(\theta)
\end{array}\right)
$$

The explicit form of the $\gamma$-matrix basis as well as the form of the Weyl spinors we will use has been given in appendix D. We will solve the Dirac equation in terms of the Weyl spinors $\Psi=\left(\Psi_{-}, \Psi_{+}\right)^{T}$.

\subsubsection{Linear equations}

Since the fermion is massless we can consider the positive and negative chirality spinors separately. To evaluate the spin connection we choose the vierbein:

$$
\begin{aligned}
e^{1} & =\sqrt{\frac{g_{2}(Y)}{2}} d Y & e^{2} & =\frac{1}{\sqrt{2 g_{2}(Y)}}(d \psi+\cos \theta d \phi) \\
e^{3} & =\sqrt{\frac{Y}{2}} d \theta & e^{4} & =\sqrt{\frac{Y}{2}} \sin \theta d \phi .
\end{aligned}
$$

Substituting our ansatz for the positive chirality wave function $\Psi_{+}=e^{i n \psi+i m \phi}\left(\begin{array}{c}G_{3}(Y) S_{3}(\theta) \\ G_{4}(Y) S_{4}(\theta)\end{array}\right)$ we find that, apart from constant factors, the Dirac equation takes the schematic form

$$
\begin{aligned}
& S_{3}(\theta)\left(L_{3}(x) \cdot G_{3}(x)\right)+G_{4}(x)\left(N_{3}(\theta) \cdot S_{4}(\theta)\right)=0 \\
& S_{4}(\theta)\left(L_{4}(x) \cdot G_{4}(x)\right)+G_{3}(x)\left(N_{4}(\theta) \cdot S_{3}(\theta)\right)=0 .
\end{aligned}
$$

There are similar equations for the negative chirality spinors. The differential operators $L_{i}$ and $N_{i}$, in this case are given by

$$
\begin{aligned}
-L_{3} & =\sqrt{\frac{Y}{g_{2}(Y)}}\left(\frac{d}{d Y}-n g_{2}(Y)+w\right)+\left(\frac{g_{2}(Y)-Y g_{2}^{\prime}(Y)}{4 \sqrt{Y g_{2}^{3}(Y)}}\right) \\
L_{4} & =\sqrt{\frac{Y}{g_{2}(Y)}}\left(\frac{d}{d Y}+n g_{2}(Y)-w\right)+\left(\frac{g_{2}(Y)-Y g_{2}^{\prime}(Y)}{4 \sqrt{Y g_{2}^{3}(Y)}}\right) \\
N_{3} & =\frac{d}{d \theta}-m \csc \theta+\left(\frac{1}{2}+n\right) \cot \theta \\
N_{4} & =\frac{d}{d \theta}+m \csc \theta+\left(\frac{1}{2}-n\right) \cot \theta .
\end{aligned}
$$


These equations are equivalent to second order equations for the radial and angular functions. As argued in appendix A, it is sufficient to focus on the solutions to the linear set of equations

$$
L_{3}(Y) \cdot G_{3}(Y)=0=L_{4}(Y) \cdot G_{4}(Y) \quad \text { and } \quad N_{3}(\theta) \cdot S_{4}(\theta)=0=N_{4}(\theta) \cdot S_{3}(\theta) .
$$

These have the following solutions:

$$
\Psi_{+}=e^{i n \psi+i m \phi}\left(\begin{array}{c}
C_{3} e^{-w Y+\frac{2 Y n}{k}}\left(\frac{8 Y}{k g_{2}(Y)}\right)^{-\frac{1}{4}+\frac{n}{2}}(\sin \theta)^{-\frac{1}{2}+n}\left(\tan \frac{\theta}{2}\right)^{-m} \\
C_{4} e^{w Y-\frac{2 Y n}{k}}\left(\frac{8 Y}{k g_{2}(Y)}\right)^{-\frac{1}{4}-\frac{n}{2}}(\sin \theta)^{-\frac{1}{2}-n}\left(\tan \frac{\theta}{2}\right)^{m}
\end{array}\right)
$$

\subsubsection{The counting of zero modes}

We must now identify the normalizable solutions. The measure factor $\sqrt{g}$ is proportional to $Y \sin \theta$. Near the tip at $Y=0$, we have that the wave-functions behave like $Y^{-\frac{1}{2} \pm n}$. The measure factor cancels the $Y^{-\frac{1}{2}}$ prefactor when we square a wave-function component. A similar phenomenon occurs for the angular factors.

We also find that for positive momentum $n$, the component $C_{4}$ needs to be zero in order to have regular wave-functions. Rewriting the third component of the wave function in terms of the argument $\frac{\theta}{2}$, we obtain

$$
\Psi_{+, 3} \sim\left(\sin \frac{\theta}{2}\right)^{-\frac{1}{2}+n-m}\left(\cos \frac{\theta}{2}\right)^{-\frac{1}{2}+n+m} .
$$

The exponent of both factors needs to be positive, up to a shift by $-1 / 2$. We conclude that $n \geq|m|$.

We moreover have that the parity of $2 n$ and of $2 m$ needs to be opposite. This is because near the tip of the space (which is locally a Euclidean four-plane) the angles $\psi$ and $\phi$ are related to angles $\xi_{1,2}$ in two two-planes through the formulas $\psi=-\xi_{1}-\xi_{2}$ and $\phi=\xi_{2}-\xi_{1}$. A $2 \pi$ rotation in one of these two-planes must give a minus sign to the fermion wave-function. All these constraints taken together imply that the quantum number $m$ lies in the window

$$
-n+1 / 2 \leq m \leq n-1 / 2,
$$

and jumps by integers when we fix $n$. For a given momentum $n$, there is therefore a degeneracy of $2 n$ arising from the two-sphere. This is the spin-degeneracy that arises from spherical harmonics with $\operatorname{spin} l=n-1 / 2$.

In order to understand the further constraints that arise from the radial part of the problem, let us recall here the radial behavior of the function $g_{2}(Y)$ :

$$
Y^{2} \stackrel{Y \rightarrow 0}{\longleftarrow} \frac{Y}{g_{2}(Y)} \stackrel{Y \rightarrow \infty}{\longrightarrow} \frac{k Y}{2} .
$$

For negative winding $w$ there are no normalizable solutions. For positive winding, the wave functions have the following asymptotic behavior:

$$
Y^{-\frac{1}{4}+\frac{n}{2}} \stackrel{Y \rightarrow 0}{\longleftarrow} \Psi_{+, 3} \stackrel{Y \rightarrow \infty}{\longrightarrow} e^{-Y\left(w-\frac{2 n}{k}\right)} Y^{-\frac{1}{4}+\frac{n}{2}} .
$$


Therefore imposing regularity and normalizability requires the momentum to be positive and in the range

$$
\frac{1}{2} \leq n \leq \frac{k w}{2}
$$

Note that we again regularize $n-k w / 2$ such that the extreme case does contribute.

For negative momentum $n<0$, we find the spin degeneracy $2|n|$ and negative winding. Only the component $\Psi_{+, 4}$ will be non-vanishing. We need to impose the inequality:

$$
\frac{k w}{2}<n \leq-\frac{1}{2}
$$

For negative chirality the zero mode solutions to the linear differential equations are given by

$$
\Psi_{-}=e^{i n \psi+i m \phi}\left(\begin{array}{c}
C_{1} e^{-w Y+\frac{2 Y n}{k}}\left(\frac{8 Y}{k g_{2}(Y)}\right)^{-\frac{1}{4}+\frac{n}{2}}(\sin \theta)^{-\frac{1}{2}-n}\left(\tan \frac{\theta}{2}\right)^{m} \\
C_{2} e^{w Y-\frac{2 Y n}{k}}\left(\frac{8 Y}{k g_{2}(Y)}\right)^{-\frac{1}{4}-\frac{n}{2}}(\sin \theta)^{-\frac{1}{2}+n}\left(\tan \frac{\theta}{2}\right)^{-m}
\end{array}\right)
$$

Noting the flip of signs in the angular wave functions relative to the positive chirality wave functions, one can check that there are no zero modes of negative chirality.

Finally, the partition sum that counts the zero modes, keeping track of the momentum and winding quantum numbers, is given by

$$
\begin{aligned}
Z_{4} & =\left(\sum_{w>0} \sum_{2 n=1,2, \ldots}^{[k w]}+\sum_{w<0} \sum_{2 n=[k w-\epsilon]}^{-\frac{1}{2}}\right) 2|n| y_{1}^{n} y_{2}^{w} \\
& =\left(\sum_{w>0} \sum_{2 n=1,2, \ldots}^{[k w]}-\sum_{w<0} \sum_{2 n=[k w-\epsilon]}^{-\frac{1}{2}}\right) 2 n y_{1}^{n} y_{2}^{w},
\end{aligned}
$$

where the double square brackets $[x]$ indicate the smallest integer smaller or equal to $x$. The final result is very similar to the index counting in Euclidean Taub-NUT. Indeed, they have in common the transverse geometry responsible for the degeneracy factor, as well as the tip region. The asymptotics of the spaces is different, yet they lead to the same index. This aspect is discussed in appendices $\mathrm{A}$ and $\mathrm{C}$.

\subsection{The six-dimensional asymptotically linear dilaton background}

We turn to the explicit construction of Dirac zero modes in the six-dimensional asymptotic linear dilaton background (i.e. the case $N=3$ of background (3.1)).

The background. The metric and dilaton are given by

$$
\begin{aligned}
d s^{2} & =\frac{g_{3}(Y)}{2} d Y^{2}+\frac{2}{9 g_{3}(Y)}\left(d \psi+3 A_{\mathrm{FS}}\right)^{2}+2 Y d s_{\mathbb{C P}^{2}}^{2} \\
\Phi & =-\frac{3 Y}{k}
\end{aligned}
$$


The connection one-form $A_{\mathrm{FS}}$ on $\mathbb{C P}^{2}$ has differential equal to the Fubini-Study curvature two-form. In appendix $\mathrm{B}$ we give a detailed description of the geometry of $\mathbb{C P}^{2}$ as well as a choice of coordinates and one-forms $e^{1,2,3,4}$. Using these, the vierbein for the sixdimensional problem are chosen to be

$$
\begin{aligned}
E^{1} & =\sqrt{\frac{g_{3}(Y)}{2}} d Y \quad E^{2}=\sqrt{\frac{2}{9 g_{3}(Y)}}\left(d \psi+3 A_{\mathrm{FS}}\right) \\
E^{3,4,5,6} & =\sqrt{2 Y} e^{1,2,3,4} .
\end{aligned}
$$

The function $g_{3}(Y)$ is explicitly given by

$$
g_{3}(Y)=\frac{36 Y^{2} e^{\frac{6 Y}{k}}}{k\left(-k^{2}+e^{\frac{6 Y}{k}}\left(k^{2}-6 k Y+18 Y^{2}\right)\right.},
$$

We choose six dimensional Weyl spinors as in appendix D. The Dirac equation for the positive chirality spinor takes the schematic form

$$
S_{i}(\theta) T_{i}(\chi) L_{i}(Y) \cdot G_{i}(Y)+G_{j}(Y) T_{j}(\chi) N_{i}(\theta) \cdot S_{j}(\theta)+G_{k}(Y) S_{k}(\theta) M_{i}(\chi) \cdot T_{k}(\chi)=0 .
$$

where the indices $i, j, k$ range over the four components of a chiral spinor. Here, $L_{i}, M_{i}$ and $N_{i}$ denote differential operators depending on the single variable in parenthesis. As in the four-dimensional background, we impose the stronger condition that $L \cdot G=N \cdot S=M \cdot T=$ 0 . The positive chirality solution is then given, up to the phase factor $e^{i n \psi+i p \varphi+i m \phi}$, by:

$$
\Psi_{+}=\left(\begin{array}{c}
\frac{C_{5}}{\sqrt{Y}} e^{\frac{3 Y n}{k}-w Y}\left(\frac{36 Y^{2}}{k g_{3}(Y)}\right)^{-\frac{1}{4}+\frac{n}{2}}(\sin \chi)^{-2+2 p}(\cos \chi)^{-\frac{1}{2}-2 p-3 n}(\sin \theta)^{-\frac{1}{2}-p}\left(\tan \frac{\theta}{2}\right)^{m} \\
\frac{C_{6}}{\sqrt{Y}} e^{\frac{3 Y n}{k}-w Y}\left(\frac{36 Y^{2}}{k g_{3}(Y)}\right)^{-\frac{1}{4}+\frac{n}{2}}(\sin \chi)^{-2-2 p}(\cos \chi)^{-\frac{1}{2}+2 p+3 n}(\sin \theta)^{-\frac{1}{2}+p}\left(\tan \frac{\theta}{2}\right)^{-m} \\
\frac{C_{7}}{Y} e^{-\frac{3 Y n}{k}+w Y}\left(\frac{36 Y^{2}}{k g_{3}(Y)}\right)^{-\frac{1}{4}-\frac{n}{2}}(\sin \chi)^{-1-2 p}(\cos \chi)^{-\frac{1}{2}+2 p+3 n}(\sin \theta)^{-\frac{1}{2}-p}\left(\tan \frac{\theta}{2}\right)^{m} \\
C_{8} e^{-\frac{3 Y n}{k}+w Y}\left(\frac{36 Y^{2}}{k g_{3}(Y)}\right)^{-\frac{1}{4}-\frac{n}{2}}(\sin \chi)^{-1+2 p}(\cos \chi)^{-\frac{1}{2}-2 p-3 n}(\sin \theta)^{-\frac{1}{2}+p}\left(\tan \frac{\theta}{2}\right)^{-m}
\end{array}\right)
$$

We summarize the asymptotics of the radial function:

$$
Y^{3} \stackrel{Y \rightarrow 0}{\longleftarrow} \frac{Y^{2}}{g_{3}(Y)} \stackrel{Y \rightarrow \infty}{\longrightarrow} \frac{k Y^{2}}{2},
$$

and note the measure factor:

$$
\sqrt{\operatorname{det} g}=\frac{1}{6} Y^{2} \cos \chi \sin \theta \sin ^{3} \chi .
$$

From the flat space limit and the fermionic nature of the target space wave-functions, we conclude that $3 n$ needs to be strictly half-integer, while $2 m$ and $2 p$ are integers of opposite parity. See appendix B for details.

For negative momentum $n$, by analyzing the radial profile near the tip, we conclude that $C_{5}=C_{6}=0$. We therefore focus on the 7 and 8 components. For the 7 component, we find that

$$
C_{7} \neq 0 \Rightarrow-p \pm m \geq \frac{1}{2} \quad \text { and } \quad 3 n+2 p \geq 0,
$$


which are contradictory, implying that $C_{7}=0$. For the 8 component, we find

$$
C_{8} \neq 0 \Rightarrow p \pm m \geq \frac{1}{2} \quad \text { and } \quad+3 n+2 p \leq 0 .
$$

We can think of the quantum number $m$ as filling out a spin $p-1 / 2$ multiplet. When we refine the counting through various quantization conditions, and combine all constraints, we find that, for the 8 component, the degeneracy of zero modes for a given value of momentum $n$ is given by

$$
D(n)=\sum_{p=1 / 2,1, \ldots}^{-\frac{3 n}{2}-\frac{1}{4}} 2 p=\sum_{r=1}^{-3 n-\frac{1}{2}} r=\frac{\left(-3 n-\frac{1}{2}\right)\left(-3 n+\frac{1}{2}\right)}{2}
$$

When we analyze the positive momentum $n$ states, we find no solutions. For the negative chirality spinor the wave function is (up to the phase factor $e^{i n \psi+i p \lambda+i m \phi}$ ) of the form:

$\Psi_{-}=\left(\begin{array}{c}C_{1} e^{\frac{3 Y n}{k}-w Y}\left(\frac{36 Y^{2}}{k g_{3}(Y)}\right)^{-\frac{1}{4}+\frac{n}{2}}(\sin \chi)^{-1-2 p}(\cos \chi)^{-\frac{1}{2}+2 p+3 n}(\sin \theta)^{-\frac{1}{2}-p}\left(\tan \frac{\theta}{2}\right)^{m} \\ \frac{C_{2}}{Y} e^{\frac{3 Y n}{k}-w Y}\left(\frac{36 Y^{2}}{k g g_{3}(Y)}\right)^{-\frac{1}{4}+\frac{n}{2}}(\sin \chi)^{-1+2 p}(\cos \chi)^{-\frac{1}{2}-2 p-3 n}(\sin \theta)^{-\frac{1}{2}+p}\left(\tan \frac{\theta}{2}\right)^{-m} \\ \frac{C_{3}}{\sqrt{Y}} e^{-\frac{3 Y n}{k}+w Y}\left(\frac{36 Y^{2}}{k g_{3}(Y)}\right)^{-\frac{1}{4}-\frac{n}{2}}(\sin \chi)^{-2+2 p}(\cos \chi)^{-\frac{1}{2}-2 p-3 n}(\sin \theta)^{-\frac{1}{2}-p}\left(\tan \frac{\theta}{2}\right)^{m} \\ \frac{C_{4}}{\sqrt{Y}} e^{-\frac{3 Y n}{k}+w Y}\left(\frac{36 Y^{2}}{k g_{3}(Y)}\right)^{-\frac{1}{4}-\frac{n}{2}}(\sin 2 \chi)^{-2-2 p}(\cos \chi)^{-\frac{1}{2}+2 p+3 n}(\sin \theta)^{-\frac{1}{2}+p}\left(\tan \frac{\theta}{2}\right)^{-m}\end{array}\right)$

One notices that the angular constraints from the third and fourth components lead to contradictory requirements on the quantum number $p$, enforcing $C_{3}=C_{4}=0$. We are then led to the constraint that the momentum $n$ be positive for negative chirality spinors and find that only the first component can be non-zero. From the angular variables we first of all find the constraints

$$
p+\frac{1}{2} \leq m \leq-p-\frac{1}{2}
$$

with $p<-\frac{1}{2}$. Once again, the variable $m$ takes values in a spin $|p|-\frac{1}{2}$ representation, leading to a $2|p|$ degeneracy factor. The positivity of the $\cos \chi$ exponent leads to the constraint

$$
p>\frac{1}{4}-\frac{3 n}{2},
$$

which gives rise to an angular degeneracy of

$$
D(n)=\sum_{p=\frac{1}{4}-\frac{3 n}{2}}^{-\frac{1}{2}} 2|p|=\sum_{r=\frac{1}{2}-3 n}^{-1}|r|=\frac{\left(3 n-\frac{1}{2}\right)\left(3 n+\frac{1}{2}\right)}{2} .
$$

From the behavior at infinity, we find some further constraints on the quantum number $n$. We get (with the same regularization as in two and four dimensions):

$$
k w<3 n \leq-\frac{3}{2} \quad \text { or } \quad k w \geq 3 n \geq \frac{3}{2} .
$$


These lead to the following expression for the final index sum:

$$
\begin{aligned}
Z_{6} & =\left(\sum_{w<0} \sum_{[[k w-\epsilon]]}^{3 n=-3 / 2}-\sum_{w>0} \sum_{3 n=3 / 2,5 / 2, \ldots}^{[[k w]]}\right) \frac{\left(3 n-\frac{1}{2}\right)\left(3 n+\frac{1}{2}\right)}{2} y_{1}^{n} y_{2}^{w} \\
& =\left(\sum_{w<0} \sum_{[[k w-\epsilon]]-1 / 2}^{m=-2}-\sum_{w>0} \sum_{m=1,2, \ldots}^{[[k w]]-1 / 2}\right) \frac{m(m+1)}{2} y_{1}^{m} y_{2}^{w} .
\end{aligned}
$$

where we identified $m=3 n-1 / 2$.

\subsection{The physics underlying the index formulas}

We would like to summarize and highlight some of the common features of the results we have obtained so far. Elementary physical reasoning will give some extra insight into the results of the explicit counting. The general form of the answer for the index of the Dirac operator, for a fixed winding sector, can be written in the form,

$$
Z_{2 N}(w)=\left\{\begin{array}{lll}
\sum_{m=M(w)}^{-1} D(m, N) y^{n} & \text { for } & w<0 \\
(-1)^{N} \sum_{m=1}^{M(w)} D(m, N) y^{n} & \text { for } & w>0
\end{array}\right.
$$

where $M(w)$ is a winding dependent bound on the summation range for the quantum number $m$. The full index is obtained by summing over all possible values of the winding $w$. The degeneracy factor $D(m, N)$ was obtained by studying the angular part of the solutions of the Dirac equation in detail. We found that

$$
D(m, N)=\left\{\begin{array}{lll}
1, & \text { for } & N=1 \\
m, & \text { for } & N=2 \\
\frac{1}{2} m(m+1) & \text { for } & N=3
\end{array}\right.
$$

In fact, there is another route to these degeneracy factors that simultaneously provides its generalization. When we study the background (3.1), and concentrate on the compact directions along the $\mathbb{C P}^{N-1}$, we can imagine performing a Kaluza-Klein reduction on the circle parametrized by $\psi$, near the tip (where there is no winding contribution to the energy). That gives rise to a gauge field $N A_{\mathrm{FS}}$ on the complex projective space, and given that our fermion has momentum $n$, which turns into an electric charge, we obtain a charged massless fermion on $\mathbb{C P}^{N-1}$ with charge-magnetic field product equal to $n N$. The Dirac zero modes on the whole space correspond to Dirac zero modes in this compact slice as well, such that we must determine the degeneracy of the lowest Landau level of this generalized quantum Hall system [19]. This degeneracy is fixed by an index theorem on $\mathbb{C P}^{N-1}$ with magnetic field [20]. The index is given by the integral over the manifold of a power of the generating (Fubini-Study) line bundle $L$ and the A-roof genus:

$$
\begin{aligned}
D\left(n N-\frac{N}{2}+1, N\right) & =\int_{\mathbb{C P}^{N-1}} \operatorname{ch}\left(L^{n N}\right) \hat{A}\left(\mathbb{C P}^{N-1}\right) \\
& =\frac{1}{(N-1) !}\left(n N-\frac{N}{2}+1\right)\left(n N-\frac{N}{2}+2\right) \ldots\left(n N-\frac{N}{2}+N-1\right) .
\end{aligned}
$$


We must identify $m=n N-N / 2+1$ and indeed find a perfect match between the degeneracy of states in the lowest Landau level and the number of Dirac zero modes. A corollary of the matching between the degeneracy and the index theorem is that we have only solutions of one chirality (at fixed winding number $w$ ). An alternative route to finding this degeneracy is through a careful analysis of representations of $\mathrm{U}(1) \times \mathrm{SU}(N-1) \subset \mathrm{SU}(N)$ [19]. Near the tip of the geometry, we effectively analyze the zero modes of a fermionic particle carrying a charge $n N$ and charged under the $N-1$ Cartan generators of the $\mathrm{SU}(N)$ isometry subgroup of $\mathbb{C P}^{N-1}$. Labeling the $\mathrm{U}(1)$ eigenvalues by non-negative $l_{i}$, (where $i \in\{1,2, \ldots, N-1\}$ ) we recall that the wave function at a given energy eigenvalue is characterized by the $\mathrm{U}(1)$ gauge field charge $n N$ and the total angular momentum $l=\sum_{i=1}^{N-1} l_{i}[21]$. For a given total angular momentum $l$ there are $\left(\begin{array}{c}l+N-2 \\ N-2\end{array}\right)$ wave functions. The angular momentum of ground states is bounded above in terms of the $\mathrm{U}(1)$ gauge charge. The upper bound is $m-1=n N-N / 2$. We have thus acquired a better understanding of the origin of the degeneracy of the Dirac zero modes, and the physics of a radial cross section near the origin.

On the other hand, the limits on the variable $m$, denoted $M(w)$, are determined by the radial problem, and in particular the coefficient of the radial exponential in the wave function. This can be obtained for the general case by studying the Dirac equation near radial infinity. In this limit, one can define a radial coordinate $\rho \approx \frac{Y}{k}$ and write the metric in the form

$$
\frac{d s^{2}}{k} \approx d \rho^{2}+\frac{R^{2}}{N^{2}}\left(d \psi+N A_{\mathrm{FS}}\right)^{2}+2 \rho d s_{\mathrm{FS}}^{2} .
$$

We have kept a general radius $R$ for later purposes. For now we have $R=1$. The gauge field is of the form

$$
A=\frac{w}{f(Y)}\left(d \psi+N A_{\mathrm{FS}}\right),
$$

where the function $f$ is given by

$$
f(Y)=\frac{N}{2} g_{N}(Y) .
$$

Writing out the Dirac equation for a charged fermion in these coordinates we find that it takes the following form for the individual components of the fermion

$$
\left[\frac{\partial}{\partial \rho} \pm \frac{1}{R}\left(i \frac{\partial}{\partial \psi}+A_{\psi}\right)\right] \Psi_{a}=0
$$

With $\psi$-momentum equal to $n$, we obtain the asymptotic solution

$$
\Psi_{a}=e^{i n \psi} e^{ \pm \frac{\rho}{R}\left(\frac{w}{f(\infty)}-n\right)} .
$$

Therefore, we find normalizable modes whenever

$$
|n|<\left|\frac{w}{f(\infty)}\right|
$$

In this section, we have $f(\infty)=N / k$. The bounds on $m=n N-N / 2+1$ therefore read:

$$
\left|m+\frac{N}{2}-1\right|<|k w| .
$$


This matches with what we obtained in previous sections. Besides an explicit description of the Dirac zero modes, we now also have an intuitive understanding of the counting formulas.

Using this improved understanding, we can generalize our result for any value of $N$. First of all, we observe that we have that $n N$ is a strict half-integer for $N$ odd, while it is an integer for $N$ even. As a consequence, the number $m$ is always integer. Using this fact, and the previous results, we find the partition sum for $N$ even:

$$
Z_{2 N}=\left(\sum_{w<0} \sum_{m=[k w-\epsilon]+1-\frac{N}{2}}^{-\frac{N}{2}+1}-\sum_{w>0} \sum_{m=1,2, \ldots}^{[k w]+1-\frac{N}{2}}\right) D\left(n N-\frac{N}{2}+1, N\right),
$$

while for $N$ odd:

$$
Z_{2 N}=\left(\sum_{w<0} \sum_{m=[[k w-\epsilon]]+1-\frac{N}{2}}^{-\frac{N}{2}+\frac{1}{2}}-\sum_{w>0} \sum_{m=1,2, \ldots}^{[[k w]]+1-\frac{N}{2}}\right) D\left(n N-\frac{N}{2}+1, N\right) .
$$

For $N$ odd, we obtain contributions of different chiralities, depending on the sign of the momentum, while for $N$ even, we obtain contributions only of a given chirality, but we need to take care of the fact that the degeneracy factor flips sign when going from positive to negative values of the momentum.

We note that the degeneracy factor is a polynomial in the quantum number $m$ of degree $N-1$. If we introduce a fugacity corresponding to the quantum number $m$, and suppose for instance that its norm is smaller than one, then the double sum over the winding and momentum will give rise to a pole in the fugacity of order $N$. We will explicitly perform such a resummation for $N=1$ in section 5 .

\section{Duality rotated asymptotically linear dilaton spaces}

In this section, we revisit the Dirac index calculation in a supergravity background which is either a background with asymptotic linear dilaton or a Euclidean Taub-NUT geometry. We will use a different technique to add a potential to the moduli space. Our logic is to start with a background which is a solution to the low-energy effective supergravity arising from (for instance) a heterotic string theory. The backgrounds only have a nontrivial metric and possibly a linear dilaton. We then perform a duality rotation on the isometric direction and an internal direction associated to a gauge field. We thus generate new backgrounds of supergravity that are of independent interest. The main point though is that the rotation introduces a gauge field in the background, which renders the counting problem for charged bound states well-defined.

\subsection{Duality rotated backgrounds}

In the first part of this section, we recall how to generate the new supergravity backgrounds through duality rotations. We consider the asymptotically linear dilaton background in (3.1) as being part of a heterotic string background. As explained in [11], based upon the results of $[12,13]$, one can supplement such a $2 N$-dimensional space-time with an 
extra chiral coordinate $X$, which allows one to introduce a background abelian gauge field. We will generate a new solution by doing an $O(2)$ transformation, although more general duality transformations are possible.

The duality rotation is most easily performed in the following way: encode the metric $G$, the gauge field $A$ and the anti-symmetric tensor $B$ into a $4 N+1 \times 4 N+1$ dimensional matrix

$$
\mathcal{M}=\left(\begin{array}{ccc}
K_{-}^{T} G^{-1} K_{-} & K_{-}^{T} G^{-1} K_{+} & -K_{-} G^{-1} A \\
K_{+}^{T} G^{-1} K_{-} & K_{+}^{T} G^{-1} K_{+} & -K_{+} G^{-1} A \\
-A^{T} G^{-1} K_{-} & -A^{T} G^{-1} K_{+} & A^{T} G^{-} 1 A
\end{array}\right) .
$$

Here $T$ denotes transposition and the auxiliary $2 N \times 2 N$ dimensional matrices $K_{ \pm}$are defined by

$$
\left(K_{ \pm}\right)_{\mu \nu}=-B_{\mu \nu}-G_{\mu \nu}-\frac{1}{4} A_{\mu} A_{\nu} \pm \eta_{\mu \nu} .
$$

Since the backgrounds we work with are Euclidean we will work with the Euclidean signature matrix $\eta=\mathbb{I}_{2 N}$. New solutions to low-energy heterotic supergravity actions are generated by performing a duality rotation

$$
\mathcal{M} \rightarrow \mathcal{M}^{\prime}=\Omega \mathcal{M} \Omega^{T}
$$

where $\Omega$ is a rotation matrix. We choose a duality rotation in an $\mathrm{SO}(2)$ subgroup:

$$
\Omega=\left(\begin{array}{ccc}
\mathbb{I}_{2 N-1} & 0 & 0 \\
0 & \gamma & \sqrt{1-\gamma^{2}} \\
0 & -\sqrt{1-\gamma^{2}} & \gamma
\end{array}\right)
$$

where $\gamma$ is a rotation parameter that takes values in $[0,1]$. The entries that are non-zero are chosen to be along one of the isometry directions of the manifold $M_{d}$ and an internal direction associated to the gauge field $A$.

The new metric, anti-symmetric tensor and gauge field can be unpackaged from the entries of the matrix $\mathcal{M}^{\prime}$. Finally, the dilaton in the original and new solution are related through the duality invariant combination

$$
e^{-2 \Phi^{\prime}} \operatorname{det} G^{\prime}=e^{-2 \Phi} \operatorname{det} G
$$

In the following, we apply these duality transformations to the asymptotically linear dilaton spaces in various dimensions.

\subsubsection{Asymptotically linear dilaton dyons}

Let us consider the effect of the duality rotation on the asymptotically linear dilaton backgrounds given in equation (3.1). We perform the $O(2)$ duality rotation involving the $\psi$ direction and an internal direction. The resultant metric and dilaton takes the following form

$$
\begin{aligned}
d s^{2} & =\frac{g_{N}(Y)}{2} d Y^{2}+\frac{N^{2} \beta^{2} g_{N}(Y)}{2 h_{N}(Y)^{2}}\left(\frac{d \psi}{\beta}+N A_{\mathrm{FS}}\right)^{2}+2 Y d s_{\mathrm{FS}}^{2} \\
e^{-2 \Phi} & =e^{\frac{2 N Y}{k}}\left(\frac{2 h_{N}(Y)}{N^{2} g_{N}(Y)}\right)^{2}
\end{aligned}
$$


where $\beta \in[0,1]$. We defined the function

$$
h_{N}(Y)=1-\beta+\frac{N^{2} \beta}{2} g_{N}(Y) .
$$

The other closed string fields that result from the duality transformation are the gauge field $A$ and the NS-NS two-form $B$ :

$$
\begin{aligned}
& A=-2 \sqrt{\frac{1-\beta}{\beta}} d \psi+\frac{2 \sqrt{(1-\beta) \beta}}{h_{N}(Y)}\left(\frac{d \psi}{\beta}+N A_{\mathrm{FS}}\right) \\
& B=\frac{N \beta(1-\beta)}{h_{N}(Y)} \frac{d \psi}{\beta} \wedge A_{\mathrm{FS}} .
\end{aligned}
$$

From the form of the rotated metric, gauge field and B-field, one can see that, if we rescale the coordinate $\psi$ by $\beta$, the new coordinate allows one to write the fibration over $\mathbb{C P}^{N-1}$ in a manner identical to the linear dilaton fibrations discussed in appendix B. In other words, the metric and gauge field of these duality rotated backgrounds differ from the background in (3.1) only in the detailed form of the radial functions that appear in the fibration. This will have important consequences below. We will once again denote the new coordinate by $\psi$ and it has periodicity $2 N \pi$. This choice of periodicity renders the duality rotated backgrounds genuinely new. We moreover drop the first, Wilson line term in the gauge field in equation (4.8) - the background remains a supergravity solution.

\subsection{Charged Dirac indices}

In the following we analyze the Dirac index of a fermion in the duality rotated metric that we generated, coupling with strength $e$ to the gauge field $A$ generated by the duality rotation. ${ }^{1}$ The Dirac equation we solve includes a coupling of the charged fermion to the gauge field:

$$
\gamma^{\mu}\left(\partial_{\mu}+\frac{1}{4} \omega_{\mu}^{a b} \gamma_{a b}-i e A_{\mu}\right) \Psi=0
$$

We discuss the asymptotic linear dilaton models for general $N$. We follow the intuitive discussion in subsection 3.4. The physics near the origin $Y=0$ is the same as before: we project onto the lowest Landau level on the projective space $\mathbb{C P}^{N-1}$. The asymptotics of the radial problem at $Y \rightarrow \infty$ is slightly different: ${ }^{2}$

$$
\begin{aligned}
\frac{d s^{2}}{k} & \approx \frac{d Y^{2}}{k^{2}}+\frac{N^{4} \beta^{2}}{k^{2} h_{N}(\infty)^{2}}\left(d \psi+N A_{\mathrm{FS}}\right)^{2}+\frac{2 Y}{k} d s_{\mathrm{FS}}^{2}, \\
e A & \approx \frac{e}{h_{N}(\infty)}\left(d \psi+N A_{\mathrm{FS}}\right) .
\end{aligned}
$$

\footnotetext{
${ }^{1}$ We will not limit ourselves to embeddings of this model in string theory. In that context, the charge $e$ would be quantized. The supersymmetric quantum mechanics model corresponding to a manifold with gauge bundle [14] can in any case be thought off as the dimensional reduction of a heterotic non-linear sigma-model.

${ }^{2}$ We rescaled the electric charge $e$ to $\frac{e}{2 \sqrt{\beta(1-\beta)}}$.
} 
If we make the identifications

$$
\begin{aligned}
w & =e \\
f(Y) & =h_{N}(Y) \\
R & =\frac{N^{2} \beta}{k h_{N}(\infty)} \quad \text { with } \quad h_{N}(\infty)=1-\beta+\frac{\beta N^{2}}{k}
\end{aligned}
$$

we see that the asymptotic analysis of the restrictions on the momentum of the bound state runs exactly as in subsection 3.4. One can thus write down the index for these backgrounds for a given value of the electric charge:

$$
Z_{2 N}(e)=\left\{\begin{array}{clr}
\sum_{n=\frac{1}{2}}^{\left[\frac{e}{h_{N}(\infty)}-\epsilon\right]} D\left(n N-\frac{N}{2}+1, N\right) y^{n} & \text { for } \quad e>0 \\
(-1)^{N} \sum_{n=\left[\frac{e}{h_{N}(\infty)}\right]}^{-\frac{1}{2}} D\left(n N-\frac{N}{2}+1, N\right) y^{n} & \text { for } \quad e<0 .
\end{array}\right.
$$

Here we have used the degeneracy function $D$ that counts the index of the Dirac operator on $\mathbb{C P}^{N-1}$ with magnetic field. For odd $N$ we have to replace the square brackets appropriately.

There are some subtle differences between this class of models and the ones we studied earlier. In particular, for the higher dimensional heterotic backgrounds it turns out that the Dirac equation for the components are not explicitly integrable in terms of known special functions. In order to illustrate these differences, we show how to proceed with the explicit calculation of the zero modes in the four-dimensional example. We confirm that the counting of zero modes leads to equation (4.12) for the case $N=2$. A similar analysis governs the case of dimension $2 N$.

\subsubsection{Four-dimensional asymptotically linear dilaton dyon}

For the calculation of the index on the four-dimensional heterotic dyon, we choose the following vierbein:

$$
\begin{aligned}
E^{1} & =\sqrt{\frac{g_{2}(Y)}{2}} d Y & E^{2} & =\frac{\sqrt{2 g_{2}(Y)} \beta}{h_{2}(Y)}(d \psi+\cos \theta d \phi) \\
E^{3} & =\sqrt{\frac{Y}{2}} d \theta & E^{4} & =\sqrt{\frac{Y}{2}} \sin \theta d \phi .
\end{aligned}
$$

The gauge field is given by

$$
e A=\frac{e}{h_{2}(Y)}(d \psi+\cos \theta d \phi) .
$$

The Dirac equation is solved as before and the positive chirality wave functions are

$$
\Psi_{+}=e^{i n \psi+i m \phi}\left(\begin{array}{c}
G_{3}(Y)(\sin \theta)^{-\frac{1}{2}+n}\left(\tan \frac{\theta}{2}\right)^{-m} \\
G_{4}(Y)(\sin \theta)^{-\frac{1}{2}-n}\left(\tan \frac{\theta}{2}\right)^{m}
\end{array}\right) .
$$

The radial wave functions satisfy the equations

$$
\frac{d G_{a}(Y)}{d Y}+W(Y) G_{a}(Y)=0 \quad \text { with } \quad a=3,4 .
$$


where

$$
\begin{aligned}
W_{3}(e, n, Y) & =\frac{e}{2 \beta}-\frac{n}{2 \beta} h_{2}(Y)+\frac{1}{4 Y}+\frac{g_{2}^{\prime}(Y)}{4 g_{2}(Y)}-\frac{k \beta g_{2}^{\prime}(Y)}{h_{2}(Y)}+\frac{(1-\beta)}{4 Y h_{2}(Y)} \\
\text { and } \quad W_{4}(e, n, Y) & =W_{3}(-e,-n, Y) .
\end{aligned}
$$

All but the last term are explicitly integrable in terms of known functions. The solution is of the form

$$
\begin{aligned}
& G_{3}(Y)=e^{\int d Y \frac{(1-\beta)}{4 Y h_{2}(Y)}} \sqrt{\frac{h_{2}(Y)}{Y}} e^{-\frac{Y}{2 \beta}\left(e-n\left(1-\beta+\frac{4 \beta}{k}\right)\right)}\left(\frac{8 Y}{k g_{2}(Y)}\right)^{\frac{1}{4}+\frac{n}{2}} \\
& G_{4}(Y)=e^{\int d Y \frac{(1-\beta)}{4 Y h_{2}(Y)}} \sqrt{\frac{h_{2}(Y)}{Y}} e^{\frac{Y}{2 \beta}\left(e-n\left(1-\beta+\frac{4 \beta}{k}\right)\right)}\left(\frac{8 Y}{k g_{2}(Y)}\right)^{\frac{1}{4}-\frac{n}{2}} .
\end{aligned}
$$

By analyzing the profile of the function $h_{2}(Y)$, one can check that the non-integrable term leads to a finite correction to the wave-function, well-behaved near the tip at $Y=0$ and near radial infinity. It is immaterial for the purposes of counting the bound states. There are no normalizable modes with negative chirality. The index partition sum is calculated precisely as before with the result:

$$
Z_{4}^{h e t}(e)=\left(\sum_{2 n=1}^{\left[\frac{2 e}{h_{2}(\infty)}-\epsilon\right]}-\sum_{2 n=\left[\frac{2 e}{h_{2}(\infty)}\right]}^{-1}\right) 2 n y^{n} .
$$

where we have identified $h_{2}(\infty)=1-\beta+\frac{4 \beta}{k}$. This indeed coincides with the formula in equation (4.12) which was derived based on general considerations.

\section{Conclusions and future directions}

In this paper, we computed the indices of supersymmetric quantum mechanical models associated to asymptotically linear dilaton spaces. We used two ways of regularizing a marginal bound state problem. Firstly, through the introduction of a potential consistent with supersymmetry and associated to a Killing isometry in target space (either through winding, or through rotating an orthogonally wound string). Secondly, through a supergravity duality transformation that introduces a gauge field, and coupling the space-time spinor to the gauge field. We calculated the indices by explicitly solving the massless Dirac equation. We argued that we can compute the indices by solving linear equations, which leads to a technical improvement on the counting of bound states for instance on Euclidean Taub-NUT with self-dual gauge field. The physical intuition behind the counting formulas is that the zero modes have a degeneracy equal to that of the lowest Landau level on $\mathbb{C P}^{N-1}$. It would be interesting to employ generalized index theorems on non-compact asymptotically linear dilaton spaces to check our results using a topological index calculation. We believe the supersymmetric quantum mechanical models that we defined and partially studied in this paper are interesting by themselves, and sufficiently versatile to find numerous applications in higher dimensional supersymmetric theories such as supersymmetric field theory or string theory. In the following, we wrap up by pointing out potential applications of our results in two specific contexts. 


\subsection{Mock modular forms}

We would like to illustrate that the quantum mechanics index calculation is sufficient to reproduce the mock modular form that features in the elliptic genus of the $N=2$ superconformal cigar conformal field theory in $1+1$ dimensions [23-26]. It is important to first understand what aspects of the conformal field theory are captured by the super quantum mechanics obtained via the Scherk-Schwarz reduction. It is well known that the $N=2$ cigar coset conformal field theory can be written as a product of free fermions and bosons. It is only the zero modes of the bosonic fields that are captured by the super quantum mechanics.

We have determined the number of space-time positive and negative chirality zeromodes of a space-time fermion. The space-time fermion can be thought of as arising from the quantization of right-moving fermionic zero-modes of the worldsheet conformal field theory, and bosonic zero modes. To obtain a space-time fermion, we would take the leftmovers in the NS sector. In what follows, we will prefer to think about Ramond-Ramond sector states in the conformal field theory. These correspond to space-time bosons, and we will accordingly shift the momentum $n$ from half-integer to integer.

The elliptic genus of the coset conformal field theory is defined as follows (see e.g. [27]):

$$
\chi_{\mathrm{cos}}=\operatorname{Tr}(-1)^{F} q^{L_{0}-\frac{c}{24}} \bar{q}^{\bar{L}_{0}-\frac{c}{24}} z^{J_{0}} y^{P},
$$

where $L_{0}$ denotes left-moving conformal dimension, $J_{0}$ left-moving R-charge, and $P$ a global U(1) charge (e.g. momentum). In order to lift the index calculation and complete it to an elliptic genus what we would like to do is to express the R-charge and conformal dimensions for both left and right moving states of the Ramond-Ramond sector in terms of the momentum and winding around the asymptotic circle $\psi$. The idea then is to assign appropriate weights (dependent on the modular parameter $q$ and the R-charge chemical potential $z$ ) to the variables $y_{1}$ and $y_{2}$ in the index result (3.15) that would allow us to read off the contribution of the quantum mechanics to the elliptic genus of the conformal field theory.

The conformal algebra of the cigar conformal field theory has been discussed in detail in for instance [22]. Since the quantum mechanics captures the contribution of the bosonic zero modes, their contribution to the conformal dimension and R-charge can be read off to be

$$
\begin{aligned}
L_{0}^{Q M}-\frac{1}{4 k} & =\frac{(n-k w)^{2}}{4 k}+\frac{p_{\mathrm{rad}}^{2}}{2} \\
J_{0}^{Q M} & =\frac{n-k w}{k} .
\end{aligned}
$$

Here $p_{\text {rad }}$ is the radial momentum along the $Y$ direction. The right moving conformal dimension is similarly given by

$$
\bar{L}_{0}^{Q M}-\frac{1}{4 k}=\frac{(n+k w)^{2}}{4 k}+\frac{p_{\text {rad }}^{2}}{2} .
$$

We now impose the right-movers to be in the ground state in order to calculate the elliptic genus; this automatically fixes the total right-moving conformal dimension $\bar{L}_{0}=\frac{c}{24}$. For 
the left movers, we have to calculate the left-moving conformal dimension $L_{0}-\frac{c}{24}$, which we can rewrite as the difference between left-moving and right-moving conformal dimension $L_{0}-\bar{L}_{0}$. Using the explicit expressions for these charges, we find that

$$
L_{0}-\bar{L}_{0}=-n w .
$$

Using this and the expression for the R-current in terms of the momentum and winding quantum numbers, we can now assign appropriate weights in order to calculate that part of the elliptic genus of the cigar conformal field theory that arises from the super quantum mechanics. ${ }^{3}$ We obtain

$$
Z_{2}=\left(\sum_{n \leq-1, n+k w \geq 0}-\sum_{n \geq 0, n+k w \leq-1}\right) z^{\frac{k w-n}{k}} q^{-n w} y^{n} .
$$

We shifted the momentum $n$ downward by $1 / 2$ to take into account the fact that we spectrally flowed from the NS sector to the R sector for the left-movers.

We shall now show that this results captures the non-trivial factor of the holomorphic part of the full elliptic genus. To show this, we start with the holomorphic contribution [23] to the cigar coset elliptic genus from [24-26]:

$$
\chi_{\mathrm{cos}, \mathrm{hol}}=\frac{1}{k} \sum_{\gamma, \delta \in \mathbb{Z}_{k}} e^{\frac{2 \pi i \gamma \delta}{k}} \frac{i \theta_{11}(\tau, \alpha)}{\eta^{3}} \sum_{m \in \mathbb{Z}} \frac{q^{\frac{(k m+\gamma)^{2}}{k}} z^{2 \frac{k m+\gamma}{k}}}{1-z^{\frac{1}{k}} q^{m+\frac{\gamma}{k}} e^{\frac{2 \pi i \delta}{k}}} y^{-(\gamma+k m)},
$$

We then follow $[24,26]$ and expand the result in a particular regime of parameters $(y, z, q)$ as a double sum:

$$
\chi_{\mathrm{cos}, \mathrm{hol}}=\frac{i \theta_{11}(\tau, \alpha)}{\eta^{3}}\left(\sum_{n \leq 0, n+k w \geq 0}-\sum_{n \geq 1, n+k w \leq-1}\right) z^{\frac{k w-n}{k}} q^{-n w} y^{n} .
$$

We see several differences between formulas (5.5) and (5.7). As mentioned earlier, in the quantum mechanics, we have not taken into account the left-moving oscillator contributions of fermions or bosons, nor the degeneracy of the left-moving worldsheet vacuum. Therefore, there is a factor $i \theta_{11}(\tau, \alpha) / \eta^{3}$ that needs to be supplemented to equation (5.5). Secondly, we see that we have taken a different scheme to divide $n=0$ zero modes into two sets. The scheme is a matter of choice. The expressions (5.5) and (5.7) differ by a theta-function that transforms well under modular and elliptic transformations.

Note also that if we now want to address the full elliptic genus problem including the non-holomorphic contributions, we need to study one more aspect of the right-moving super quantum mechanics. Indeed, the non-holomorphic contribution contains a measure factor arising from the difference in the density of states of primary right-moving bosons and fermions. To determine this difference, it is sufficient to know the asymptotic form of the supercharge of the supersymmetric quantum mechanics. It takes the form:

$$
\tilde{Q} \approx\left(i p_{\text {rad }}+n+k w\right) \tilde{\psi},
$$

\footnotetext{
${ }^{3}$ The sign of the momentum is conventional. We have flipped it here for easier comparison with [24, 26]. We restrict in this section to integer levels $k$.
} 
where $p_{\text {rad }}$ is the radial momentum. We can then indeed check that the measure factor, determined in [24] and discussed in detail in [26] is given by the inverse of the asymptotic expression of the supercharge (stripped off the worldsheet fermion operator $\tilde{\psi}$ ). The underlying reason for this is that the asymptotic supercharge relates asymptotic bosonic and fermionic wave-functions, and therefore also dictates the ratio between bosonic and fermionic reflection amplitudes. The latter in turn codes the difference in densities of states [30].

Therefore, we see that in the two-dimensional cigar background, the class of supersymmetric quantum mechanical models that we considered captures all of the non-trivial data necessary to reconstitute the full elliptic genus of the two-dimensional superconformal field theory, namely both the Appell-Lerch sum, and its modular completion.

It will be interesting to apply the same idea to the higher dimensional target spaces at our disposal and to generate new examples of non-compact elliptic genera containing mock modular forms. The literature on physical models for mock modular forms is growing (see e.g. [31-35] for recent applications). One would also like to generate models for more exotic or new mock modular forms. The higher dimensional examples we have are less easily lifted to full conformal field theory results, amongst other reasons because asymptotically the $\psi$ circle remains fibered. Their degeneracy factors do give rise to poles of order $N$ when we re-sum but a further twist of the model may be needed to render the degeneracies chiral on the worldsheet. One goal could be to obtain a conformal field theory model for the interesting double pole Appell-Lerch sums with mock modular behavior that have been identified in [34] in the context of D-brane bound state counting with application to the entropy of supersymmetric black holes.

\subsection{Counting domain wall bound states}

Euclidean Taub-NUT arises as the moduli space of monopoles in supersymmetric YangMills theory $[16,17]$ and the low energy dynamics is determined by geodesic motion on the moduli space. A potential or self-dual gauge field arises when we have at least a rank two gauge group, and misaligned vacuum expectation values for adjoint scalars [36]. This leads to the application of index counting on Euclidean Taub-NUT space [5] to the counting of monopole bound states [6] as well as to the counting of D-brane bound states [7].

Interestingly it was shown in [37] that the cigar background arises as the moduli space of domain walls in a three dimensional U(1) gauge theory with 8 supercharges. The simplest setting is when the theory admits three isolated vacua, which happens when we have three charged hypermultiplets with distinct masses (and judicious choices for the signs of FayetIliopoulos parameters). The domain walls between the three distinct vacua preserve $\mathcal{N}=$ $(2,2)$ supersymmetry and their low energy dynamics is described by motion on a moduli space with cigar shaped target (when we ignore the center of mass mode). In the infrared, the dynamics is described by the cigar conformal field theory (or its mirror, if we approach the dynamics through a calculation of the Liouville interaction potential) [37]. This system can also be realized in string theory in terms of D2-branes inside, and interpolating between, parallel D6-branes in the presence of a background NSNS two-form. 
It is an interesting question to explore the realization of higher dimensional asymptotically linear dilaton spaces as moduli spaces of (e.g. more numerous) domain walls. Our index calculations would then count domain wall bound states.

\section{Acknowledgments}

We would like to thank Ghanshyam Date, Justin David, Ashoke Sen, Nemani Suryanarayana and especially Atish Dabholkar and Sameer Murthy for useful discussions. Our research is partly funded by the grant ANR-09-BLAN-0157-02.

\section{A First order differential equations}

In this section we give an argument for the fact that in the models we consider, it is sufficient to solve linear differential equations when looking for zero modes of the twisted Dirac operator. We start out with a four-dimensional metric

$$
d s^{2}=\frac{g(r)}{2} d r^{2}+\frac{1}{2 g(r)}(d \psi+\cos \theta d \phi)^{2}+\frac{f(r)}{2}\left(d \theta^{2}+\sin ^{2} \theta d \phi^{2}\right)
$$

and gauge field

$$
A=\frac{1}{2 g(r)}(d \psi+\cos \theta d \phi)
$$

We choose a vierbein of the form

$$
e^{1}=\sqrt{\frac{g(r)}{2}} d r, e^{2}=\frac{1}{\sqrt{2 g(r)}}(d \psi+\cos \theta d \phi), e^{3}=\sqrt{\frac{f(r)}{2}} d \theta, e^{4}=\sqrt{\frac{f(r)}{2}} \sin \theta d \phi .
$$

The resulting radial and angular differential operators entering the massless Weyl fermion equation

$$
\begin{aligned}
& S_{3}(\theta)\left(L_{3}(r) \cdot G_{3}(r)\right)+G_{4}(r)\left(N_{3}(\theta) \cdot S_{4}(\theta)\right)=0 \\
& S_{4}(\theta)\left(L_{4}(r) \cdot G_{4}(r)\right)+G_{3}(r)\left(N_{4}(\theta) \cdot S_{3}(\theta)\right)=0,
\end{aligned}
$$

are:

$$
\begin{aligned}
-L_{3} & =\sqrt{\frac{f}{g}}\left(\frac{d}{d r}-n g+\frac{e}{2}\right)+\left(\frac{g\left(2 f^{\prime}-1\right)-f g^{\prime}}{4 \sqrt{f g^{3}}}\right) \\
L_{4} & =\sqrt{\frac{f}{g}}\left(\frac{d}{d r}+n g-\frac{e}{2}\right)+\left(\frac{g\left(2 f^{\prime}-1\right)-f g^{\prime}}{4 \sqrt{f g^{3}}}\right) \\
N_{3} & =\frac{d}{d \theta}-m \csc \theta+\left(\frac{1}{2}+n\right) \cot \theta \\
N_{4} & =\frac{d}{d \theta}+m \csc \theta+\left(\frac{1}{2}-n\right) \cot \theta .
\end{aligned}
$$

These equations are equivalent to second order equations for the radial and angular functions which read as follows:

$$
\begin{aligned}
& N_{4} N_{3} S_{4}=\lambda S_{4} \\
& L_{3} L_{4} G_{4}=\lambda G_{4},
\end{aligned}
$$


where $\lambda$ is a generic eigenvalue. The operators $N_{3,4}$ are anti-hermitian conjugate with respect to the measure $\sin \theta$. The operators $L_{3,4}$ are hermitian conjugate with respect to the measure $d r f^{1 / 2} \exp \left(-\frac{1}{2} \int^{r} f^{-1}\right)$. For example, the measure in the Euclidean TaubNUT example is $d x(x+1)$ and in the four-dimensional asymptotically linear dilaton solution it is simply $d Y$. Assuming these measures on our space of wave-functions, we find that the angular problem implies that $\lambda \leq 0$ while the radial problem requires $\lambda \geq 0$, thus proving that necessarily $\lambda=0$. In turn, this implies that $N_{3} S_{4}=0$ and $L_{4} G_{4}=0$. Thus, we can restrict to solving the linear differential equations.

Whether the measure we chose is appropriate is a more subtle matter. Near radial infinity, the wave functions behave exponentially, and any polynomial measure will lead to the same conclusion about normalizability. Near the origin though, the polynomial measure matters, but only for the smallest quantum numbers. For those, we assume that the pattern we find for the indices at large quantum numbers persists. In cases where one can solve explicitly the second order differential equation, one can check the validity of our approach even for small quantum numbers. For example for Euclidean Taub-NUT, we can prove explicitly the validity of our approach by comparing our results with those of Pope [5]. This is reviewed in appendix C.

More intuitively, the reduction to $\lambda=0$ is equivalent to a projection onto the lowest Landau level of a charged fermion on $\mathbb{C P}^{N-1}$ with magnetic field.

Remark on asymptotics. For future purposes, we note an interesting aspect of the first order differential operators (A.4). The angular operators $N_{3,4}$ turn out to be independent of the $\mathbb{C P}^{1}$ warp factor $f(r)$, while the only dependence of the radial operators $L_{3,4}$ on the metric factor $f(r)$ is encoded in the term $\frac{2 f^{\prime}-1}{4 f}$. To analyze the asymptotic behavior of the fermionic wave function and ascertain the constraints on the quantum numbers in the counting formula from the asymptotics (as in subsection 3.4), we can solve the first order system after taking the limit on the functions $g(r)$ and $f(r)$ in the differential operators. Consequently, two backgrounds with the same asymptotic behavior of $g(r)$ and with the asymptotic value of $\frac{2 f^{\prime}-1}{4 f}$ differing by a subleading power of the radial coordinate, will support the same fermionic zero modes. The index will be identical though the backgrounds differ. This happens in the case for the counting in Euclidean Taub-NUT done in appendix $\mathrm{C}$ and the four-dimensional asymptotically linear dilaton background analyzed in the main text.

\section{B Fibrations over $\mathbb{C P}^{N-1}$}

\section{B.1 The sphere as a circle fibration}

Let us consider the metric on an odd dimensional sphere $S^{2 N-1}$. We use a simple embedding of the sphere in $\mathbb{C}^{N}$, parametrized by the $N$ complex coordinates $z_{a}$ where

$$
z_{a}=\mu_{a} e^{i \xi_{a}} .
$$

The $\mu_{a}$ satisfy the constraint $\sum_{a} \mu_{a}^{2}=1$. The metric on $S^{2 N-1}$ is given by

$$
d s_{S^{2 N-1}}^{2}=\sum_{a=1}^{N} d \mu_{a}^{2}+\sum_{a=1}^{N} \mu_{a}^{2} d \xi_{a}^{2} .
$$


In the patch where $\mu_{1} \neq 0$, inhomogeneous coordinates on $\mathbb{C} \mathbb{P}^{N-1}$ are given by

$$
Z_{i}=\frac{\mu_{i}}{\mu_{1}} e^{i \xi_{i 1}} \quad \text { for } \quad i \in\{2, \ldots, N\}
$$

where $\xi_{i 1}=\xi_{i}-\xi_{1}$. The Fubini-Study metric and the associated connection one-form is given by

$$
d s_{\mathrm{FS}}^{2}=\left(1+\left|Z_{2}\right|^{2}+\ldots+\left|Z_{N}\right|^{2}\right)^{-1} \sum_{i, j=2}^{N}\left(\delta_{i j}-\frac{Z_{i} \bar{Z}_{j}}{1+\left|Z_{2}\right|^{2}+\ldots+\left|Z_{N}\right|^{2}}\right) d \bar{Z}_{i} d Z_{j}
$$

Substituting the expression for the coordinates $Z_{i}$ into the Fubini-Study metric, we find that the metric on the projective space is given by

$$
d s_{\mathrm{FS}}^{2}=\sum_{a=1}^{N} d \mu_{a}^{2}+\sum_{i=2}^{N} \mu_{i}^{2}\left(d \xi_{i 1}\right)^{2}-\left(\sum_{i=2}^{N} \mu_{i}^{2} d \xi_{i 1}\right)^{2}
$$

Subtracting the two expressions, and using the following identity:

$$
\sum_{a} \mu_{a}^{2} d \xi_{a}^{2}-\left(\sum_{a} \mu_{a}^{2} d \xi_{a}\right)^{2}-\left(\sum_{i=2}^{N} \mu_{i}^{2}\left(d \xi_{i 1}\right)^{2}-\left(\sum_{i=2}^{N} \mu_{i}^{2} d \xi_{i 1}\right)^{2}\right)=0
$$

we find the simple relation between the sphere metric and the metric on projective space:

$$
d s_{S^{2 N-1}}^{2}=d s_{\mathrm{FS}}^{2}+\left(\sum_{a} \mu_{a}^{2} d \xi_{a}\right)^{2} .
$$

We can interpret the second term on the right as a Hopf fibration over $\mathbb{C P}^{N-1}$ as follows. The connection one-form on projective space, whose differential is the Kähler form, is given by

$$
A_{\mathrm{FS}}=-\frac{i}{2}\left(1+\left|Z_{2}\right|^{2}+\ldots+\left|Z_{N}\right|^{2}\right)^{-1} \sum_{i=2}^{N}\left(Z_{i} d \bar{Z}_{i}-\bar{Z}_{i} d Z_{i}\right) .
$$

In terms of the $\left(\mu_{i}, \xi_{i 1}\right)$ coordinates, we find that

$$
A_{\mathrm{FS}}=-\sum_{i=2}^{N} \mu_{i}^{2} d \xi_{i 1}=d \xi_{1}-\sum_{a=1}^{N} \mu_{a}^{2} d \xi_{a}
$$

In other words, the one-form, whose square equals the difference between the sphere metric and the projective space metric is given by

$$
\sum_{a=1}^{N} \mu_{a}^{2} d \xi_{a}=d \xi_{1}-A_{\mathrm{FS}}
$$

One can also write this in a more symmetric form as follows:

$$
\begin{aligned}
\sum_{a=1}^{N} \mu_{a}^{2} d \xi_{a} & =\frac{d \xi_{1}+d \xi_{2}+\ldots d \xi_{N}}{N}+\sum_{i=2}^{N}\left(\mu_{i}^{2}-\frac{1}{N}\right) d \xi_{i 1} \\
& =-d \tilde{\psi}-\sum_{a=1}^{N}\left(\mu_{a}^{2}-\frac{1}{N}\right)\left(d \xi_{a}-d \tilde{\psi}\right) \\
& =-d \tilde{\psi}-\tilde{A}_{\mathrm{FS}}
\end{aligned}
$$


where we have defined $\tilde{\psi}=-\frac{1}{N} \sum_{a=1}^{N} \xi_{a}$ and used $\xi_{a}-\tilde{\psi}$ to parametrize the angles on $\mathbb{C P}^{N-1}$. We can define the fibre coordinate to be $\tilde{\psi}$ as defined above or use $\xi_{1}$ as in (B.10). In either case what we have shown is the useful link between the sphere and the projective space metrics:

$$
d s_{S^{2 N-1}}^{2}=\left(d \tilde{\psi}+A_{\mathrm{FS}}\right)^{2}+d s_{\mathrm{FS}}^{2} .
$$

\section{B.2 Asymptotically linear dilaton fibrations}

The metric of the general asymptotically linear dilaton theory is given by

$$
d s^{2}=\frac{g_{N}(Y)}{2} d Y^{2}+\frac{2}{g_{N}(Y)}\left(\frac{d \psi}{N}+A_{\mathrm{FS}}\right)^{2}+2 Y d s_{\mathrm{FS}}^{2},
$$

The important observation that links our discussion of the circle fibration to these metrics is the behavior of the metric near the origin $Y \rightarrow 0$. In this limit we have

$$
d s^{2} \approx \frac{d Y^{2}}{2 Y}+2 Y\left[\left(\frac{d \psi}{N}+A_{\mathrm{FS}}\right)^{2}+d s_{\mathrm{FS}}^{2}\right]
$$

Comparing, we find that we see that the metric near the tip smoothly reduces to the flat space metric if we identify the angles $\xi_{1}=-\frac{1}{N} \psi$. This fixes the periodicity of the angle $\psi$ to be $2 \pi N$ since the angle $\xi_{1}$ in a complex two-plane has periodicity $2 \pi$.

\section{B.2.1 Asymptotic linear dilaton fibration over $\mathbb{C P}^{1}$}

In the four-dimensional setting, we make the following choice of coordinates on the threesphere

$$
z_{1}=\mu_{1} e^{i \xi_{1}} \quad z_{2}=\mu_{2} e^{i \xi_{2}}
$$

where

$$
\mu_{1}=\cos \frac{\theta}{2} \quad \mu_{2}=\sin \frac{\theta}{2} .
$$

The corresponding inhomogeneous coordinate on $\mathbb{C P}^{1}$ is given by the ratio:

$$
Z=\tan \frac{\theta}{2} e^{i \xi_{21}}
$$

The Fubini-Study metric is

$$
d s_{\mathrm{FS}}^{2}=\frac{d Z d \bar{Z}}{\left(1+|Z|^{2}\right)^{2}}=\frac{1}{4}\left(d \theta^{2}+\sin ^{2} \theta d \xi_{21}^{2}\right) .
$$

It coincides with the round metric on the two sphere. The connection one-form associated to the projective space is given by

$$
A_{\mathrm{FS}}=-\frac{i}{2} \frac{Z d \bar{Z}-\bar{Z} d Z}{\left(1+|Z|^{2}\right)}=-\sin ^{2} \frac{\theta}{2} d \xi_{21}
$$

Up to a Wilson line, we can also write

$$
A_{\mathrm{FS}}=\frac{1}{2} \cos \theta d \phi .
$$


The four dimensional asymptotically linear dilaton metric we use is [4]

$$
d s^{2}=\frac{g_{2}(Y)}{2} d Y^{2}+\frac{1}{2 g_{2}(Y)}(d \psi+\cos \theta d \phi)^{2}+\frac{Y}{2}\left(d \theta^{2}+\sin ^{2} \theta d \phi^{2}\right) .
$$

The change in Wilson line above, is equivalent to a redefinition of the angle $\psi$.

\section{B.2.2 Asymptotic linear dilaton fibration over $\mathbb{C P}^{2}$}

In the six-dimensional asymptotic linear dilaton background, we make the choice of coordinates:

$$
\begin{aligned}
z_{i} & =\mu_{i} e^{i \xi_{i}} \quad i=1,2,3 \quad \text { with } \\
\mu_{1} & =\cos \chi \quad \mu_{2}=\sin \chi \cos \frac{\theta}{2} \quad \text { and } \quad \mu_{3}=\sin \chi \sin \frac{\theta}{2} .
\end{aligned}
$$

We choose the following inhomogeneous coordinates on $\mathbb{C P}^{2}[15]$ :

$$
Z_{1}=\tan \chi \cos \frac{\theta}{2} e^{i \frac{\varphi+\phi}{2}} \quad \text { and } \quad Z_{2}=\tan \chi \sin \frac{\theta}{2} e^{i \frac{\varphi-\phi}{2}} .
$$

The connection one-form takes the form

$$
A_{\mathrm{FS}}=-\frac{1}{2} \sin ^{2} \chi(d \varphi+\cos \theta d \phi) .
$$

The Fubini-Study metric is:

$$
d s_{\mathrm{FS}}^{2}=d \chi^{2}+\frac{1}{4} \sin ^{2} \chi \cos ^{2} \chi(d \varphi+\cos \theta d \phi)^{2}+\frac{1}{4} \sin ^{2} \chi\left(d \theta^{2}+\sin ^{2} \theta d \phi^{2}\right) .
$$

A vierbein for $\mathbb{C P}^{2}$ is therefore given by

$$
\begin{array}{ll}
e^{1}=d \chi & e^{2}=\frac{1}{2} \sin \chi \cos \chi(d \varphi+\cos \theta d \phi) \\
e^{3}=\frac{\sin \chi}{2} d \theta & e^{4}=\frac{1}{2} \sin \chi \sin \theta d \phi .
\end{array}
$$

The six-dimensional asymptotically linear dilaton spaces are fibrations of the form (B.13) with $N=3$ :

$$
d s^{2}=\frac{g_{3}(Y)}{2} d Y^{2}+\frac{2}{g_{3}(Y)}\left(\frac{d \psi}{3}+A_{\mathrm{FS}}\right)^{2}+2 Y d s_{\mathrm{FS}}^{2},
$$

\section{Euclidean Taub-NUT backgrounds}

In this appendix we perform the fermionic zero mode counting in the Euclidean Taub-NUT background with self-dual gauge field. The calculation of zero modes for this background has been carried out in [5]. We will revisit the calculation and arrive at the same result in a mildly more efficient manner. We moreover extend the calculation to the case of the heterotic Taub-NUT dyon. 


\section{C.1 Euclidean Taub-NUT with self-dual gauge field}

The metric of the Euclidean Taub-NUT space is given by

$$
d s^{2}=\frac{r+M}{r-M} d r^{2}+4 M^{2} \frac{r-M}{r+M}(d \psi+\cos \theta d \phi)^{2}+\left(r^{2}-M^{2}\right)\left(d \theta^{2}+\sin ^{2} \theta d \phi^{2}\right),
$$

where $M$ is a parameter with the dimension of mass. This can be put in the form (A.1) by using the radial variable $x=\frac{r-M}{2 M}$ :

$$
\frac{d s^{2}}{4 M^{2}}=\frac{(x+1)}{x} d x^{2}+\frac{x}{x+1}(d \psi+\cos \theta d \phi)^{2}+x(x+1)\left(d \theta^{2}+\sin ^{2} \theta d \phi^{2}\right) .
$$

We can set $f(x)=x(x+1), g(x)=\frac{x+1}{x}$ and $M^{2}=\frac{1}{8}$. For easier comparison with the results of [5], we will keep $M$ arbitrary in what follows. We have a self-dual gauge field given by

$$
A=-P \frac{x}{x+1}(d \psi+\cos \theta d \phi) .
$$

We note that this gauge field is proportional to the dual of the Killing vector $\partial_{\psi}$ and thus fits the analysis of section 2. The massless Dirac equation can be solved as in the examples discussed in the main text. The relevant linear differential operators that act on the positive chirality spinor components are given by

$$
\begin{aligned}
-L_{3} & =x \frac{d}{d x}+e P x-(1+x) n+\frac{(1+2 x)}{1+x}, & & L_{4}=x \frac{d}{d x}-e P x+(1+x) n+\frac{(1+2 x)}{1+x} \\
N_{3} & =\frac{d}{d \theta}-m \csc \theta+\left(\frac{1}{2}+n\right) \cot \theta, & & N_{4}=\frac{d}{d \theta}+m \csc \theta+\left(\frac{1}{2}-n\right) \cot \theta .
\end{aligned}
$$

We solve the equations $L_{3}(x) \cdot G_{3}(x)=0$ and $N_{4}(\theta) \cdot S_{3}(\theta)=0$ (and similarly for the other components) in order to find the normalizable zero modes. This is more efficient than turning to the second order differential equation as in [5]. The solutions take the form

$$
\Psi_{+}=e^{i n \psi+i m \phi}\left(\begin{array}{c}
e^{x(-e P+n)} x^{-\frac{1}{2}+n}(1+x)^{-\frac{1}{2}}(\sin \theta)^{-\frac{1}{2}+n}\left(\tan \frac{\theta}{2}\right)^{-m} \\
e^{x(e P-n)} x^{-\frac{1}{2}-n}(1+x)^{-\frac{1}{2}}(\sin \theta)^{-\frac{1}{2}-n}\left(\tan \frac{\theta}{2}\right)^{m}
\end{array}\right)
$$

The wave functions agree with [5]. For positive $e P$, we therefore find normalizable solutions for all integers $l$ which satisfy the conditions

$$
2|n|=2 l+1 \leq 2[e P] .
$$

There are no normalizable solutions with the opposite chirality. The counting function for positive $e P$ can be summarized as:

$$
Z_{\mathrm{ETN}}=\sum_{2 n=1,2, \ldots}^{2[e P]} 2 n y^{n}
$$

From subsection 3.4 (and the remark on radial asymptotics in appendix A), it should be clear that the physics underlying the counting function can be summarized by saying that we restrict to the lowest Landau level on a $\mathbb{C P}^{1}$ slice near the tip, that the degeneracy of the level is determined by its spin and that the bound state momentum is restricted by radial asymptotic normalizability. 


\section{C.2 The heterotic Euclidean Taub-NUT dyon}

In this subsection, we review the construction of the heterotic Euclidean Taub-NUT dyon, and calculate the Dirac index in this background.

\section{C.2.1 The background}

The starting point for the construction of the heterotic Taub-NUT dyon [11] is the TaubNUT metric and a constant dilaton which gives rise to a solution of low-energy heterotic string theory:

$$
d s^{2}=\frac{d r^{2}}{f_{1}(r)}+4 M^{2} f_{1}(r)(d \psi+\cos \theta d \phi)^{2}+\left(r^{2}-M^{2}\right)\left(d \theta^{2}+\sin ^{2} \theta d \phi^{2}\right)
$$

where

$$
f_{1}(r)=\frac{r-M}{r+M}
$$

As in the bulk of the paper, we use the $\mathrm{SO}(2)$ duality symmetry of the space of supergravity solutions to construct a dyonic generalization of the Taub-NUT metric [11]. The dyonic Taub-NUT metric is given by

$$
d s^{2}=\frac{d r^{2}}{f_{1}(r)}+4 M^{2} \beta^{2} \frac{f_{1}(r)}{f_{2}(r)^{2}}(d \psi+\cos \theta d \phi)^{2}+\left(r^{2}-M^{2}\right)\left(d \theta^{2}+\sin ^{2} \theta d \phi^{2}\right),
$$

where the function $f_{2}$ is

$$
f_{2}=\frac{r+(2 \beta-1) M}{r+M} .
$$

We prefer to work in with the coordinate $x=\frac{r-M}{2 M}$, in terms of which the metric takes the form

$$
\frac{d s^{2}}{4 M^{2}}=\frac{1+x}{x} d x^{2}+\frac{x(1+x)}{(x+\beta)^{2}} \beta^{2}(d \psi+\cos \theta d \phi)^{2}+x(x+1)\left(d \theta^{2}+\sin ^{2} \theta d \phi^{2}\right) .
$$

The duality rotation also induces other background fields. These are given by

$$
\begin{aligned}
B_{\psi \phi} & =4 M^{2} \frac{(1-\beta) x}{x+\beta} \cos \theta & e^{-2 \Phi} & =f_{2}(x)=\frac{x+\beta}{x+1} \\
A_{\psi} & =8 M^{2} \frac{\sqrt{\beta(1-\beta)}}{x+\beta} & A_{\phi} & =-8 M^{2} \frac{\sqrt{\beta(1-\beta)} x}{x+\beta} \cos \theta .
\end{aligned}
$$

The gauge field components can be combined to write

$$
A=8 M^{2} \sqrt{\frac{1-\beta}{\beta}}\left(d \psi-\frac{x}{x+\beta}(d \psi+\beta \cos \theta d \phi)\right) .
$$

We will neglect the first (pure gauge) term in the analysis of the Dirac equation. In order for the metric to be non-singular near the tip of the space, we impose the periodicity condition $\psi \equiv \psi+4 \pi$. This turns the duality rotated background into a genuinely new solution. Indeed, a continuous duality transformation, supplemented with new discrete identifications in the rotated solution, leads to a physically distinct background. 


\section{C.2.2 Dirac index for a charged spinor on the Taub-NUT dyon}

In this subsection, we calculate the Dirac index for a charged spinor in the Euclidean Taub-NUT dyon background. By choosing the orthonormal real vierbeins

$$
\begin{aligned}
e^{1} & =\frac{2 M d x}{\sqrt{f_{1}(x)}} & e^{2} & =2 M \beta \frac{\sqrt{f_{1}(x)}}{f_{2}(x)}(d \psi+\cos \theta d \phi) \\
e^{3} & =2 M \sqrt{x(x+1)} d \theta & e^{4} & =2 M \sqrt{x(x+1)} \sin \theta d \phi,
\end{aligned}
$$

one can check that the field strength can be written as

$$
F=\frac{2 \sqrt{\beta(1-\beta)}}{(x+1)(x+\beta)}\left(e^{3} \wedge e^{4}-e^{1} \wedge e^{2}\right) .
$$

The electromagnetic field $F$ carries electric and magnetic charge and behaves like $F_{x \psi} \approx$ $Q / x^{2}$ and $F_{\theta \phi} \approx Q \sin \theta$ at $x \rightarrow \infty$, where

$$
Q=8 M^{2} \sqrt{\beta(1-\beta)} .
$$

A gauge transformation brings the gauge field in equation (C.13) into the form

$$
A=-\frac{Q x}{x+\beta}(d \psi+\cos \theta d \phi) .
$$

The component Dirac equations can be solved as before by separating variables. The positive chirality wave functions are given by

$$
\Psi_{+}=e^{i n \psi+i m \phi} \frac{(x+\beta)^{\frac{1}{4}}}{(1+x)^{\frac{3}{4}}}\left(\begin{array}{l}
e^{\frac{x}{\beta}(e Q+n)}(\sin \theta)^{-\frac{1}{2}+n}\left(\tan \frac{\theta}{2}\right)^{-m} \\
e^{-\frac{x}{\beta}(e Q+n)}(\sin \theta)^{-\frac{1}{2}-n}\left(\tan \frac{\theta}{2}\right)^{m}
\end{array}\right) .
$$

The index generating function now equals:

$$
Z_{\mathrm{ETN}}^{\mathrm{het}}=\sum_{2 n=1,2, \ldots}^{[-2 e Q]} 2 n y^{n}
$$

for $e Q<0$.

\section{Conventions for spinors}

In two dimensions, we use the basis of gamma-matrices

$$
\gamma^{1}=\sigma_{1}=\left(\begin{array}{ll}
0 & 1 \\
1 & 0
\end{array}\right) \quad \gamma^{2}=\sigma^{2}=\left(\begin{array}{cc}
0 & -i \\
i & 0
\end{array}\right) \quad \gamma^{3}=i \gamma^{1} \gamma^{2}=-\sigma_{3}=\left(\begin{array}{cc}
-1 & 0 \\
0 & 1
\end{array}\right) .
$$

In four dimensions, we choose a Weyl basis for the gamma matrices:

$$
\gamma^{k}=\left(\begin{array}{cc}
0 & \sigma^{k} \\
-\sigma^{k} & 0
\end{array}\right) \quad \gamma^{4}=\left(\begin{array}{cc}
0 & \mathbb{I}_{2} \\
\mathbb{I}_{2} & 0
\end{array}\right) \quad \gamma^{5}=\gamma^{1} \gamma^{2} \gamma^{3} \gamma^{4}=\left(\begin{array}{cc}
-\mathbb{I}_{2} & 0 \\
0 & \mathbb{I}_{2}
\end{array}\right) .
$$


Our ansatz for the positive chirality and negative chirality spinors in four dimensions is given by:

$$
\Psi=\left(\begin{array}{l}
\Psi_{-} \\
\Psi_{+}
\end{array}\right)
$$

where the two component Weyl spinors are given by

$$
\Psi_{-}=e^{i n \psi+i m \phi}\left(\begin{array}{l}
G_{1}(Y) S_{1}(\theta) \\
G_{2}(Y) S_{2}(\theta)
\end{array}\right) \quad \text { and } \quad \Psi_{+}=e^{i n \psi+i m \phi}\left(\begin{array}{l}
G_{3}(Y) S_{4}(\theta) \\
G_{3}(Y) S_{4}(\theta)
\end{array}\right)
$$

Finally, in six dimensions we construct the gamma matrices by tensoring the four dimensional gamma matrices with two-dimensional Pauli matrices as follows:

$$
\begin{array}{lll}
\Gamma^{1}=\gamma^{1} \otimes \sigma_{3} & \Gamma^{2}=\gamma^{2} \otimes \sigma_{3} & \Gamma^{3}=\gamma^{3} \otimes \sigma_{3} \\
\Gamma^{4}=\gamma^{4} \otimes \sigma_{3} & \Gamma^{5}=\mathbb{I}_{4} \otimes \sigma_{1} & \Gamma^{6}=\mathbb{I}_{4} \otimes \sigma_{2} .
\end{array}
$$

The matrix $\Gamma^{7}$ is determined by $\sqrt{-1}$ times the product of all the gamma matrices. After permuting the third and seventh and first and fifth rows and columns, the matrix $\Gamma^{7}$ takes the canonical form:

$$
\Gamma^{7}=\left(\begin{array}{cc}
-\mathbb{I}_{7} & 0 \\
0 & \mathbb{I}_{7}
\end{array}\right)
$$

In this Weyl basis, the Dirac spinor is of the form

$$
\Psi=\left(\begin{array}{l}
\Psi_{-} \\
\Psi_{+}
\end{array}\right)
$$

where the four component Weyl spinors in six dimensions are

$$
\Psi_{-}=e^{i n \psi+i p \varphi+i m \phi}\left(\begin{array}{l}
G_{1}(Y) T_{1}(\chi) S_{1}(\theta) \\
G_{2}(Y) T_{2}(\chi) S_{2}(\theta) \\
G_{3}(Y) T_{3}(\chi) S_{3}(\theta) \\
G_{4}(Y) T_{4}(\chi) S_{4}(\theta)
\end{array}\right) \text { and } \Psi_{+}=e^{i n \psi+i p \varphi+i m \phi}\left(\begin{array}{l}
G_{5}(Y) T_{5}(\chi) S_{5}(\theta) \\
G_{6}(Y) T_{6}(\chi) S_{6}(\theta) \\
G_{7}(Y) T_{7}(\chi) S_{7}(\theta) \\
G_{8}(Y) T_{8}(\chi) S_{8}(\theta)
\end{array}\right) .
$$

Open Access. This article is distributed under the terms of the Creative Commons Attribution License which permits any use, distribution and reproduction in any medium, provided the original author(s) and source are credited.

\section{References}

[1] L. Álvarez-Gaumé and D.Z. Freedman, Potentials for the Supersymmetric Nonlinear б-model, Commun. Math. Phys. 91 (1983) 87 [InSPIRE].

[2] E. Kiritsis, C. Kounnas and D. Lüst, A Large class of new gravitational and axionic backgrounds for four-dimensional superstrings, Int. J. Mod. Phys. A 9 (1994) 1361 [hep-th/9308124] [INSPIRE].

[3] K. Hori and A. Kapustin, Duality of the fermionic $2 d$ black hole and $N=2$ Liouville theory as mirror symmetry, JHEP 08 (2001) 045 [hep-th/0104202] [INSPIRE]. 
[4] K. Hori and A. Kapustin, World sheet descriptions of wrapped NS five-branes, JHEP 11 (2002) 038 [hep-th/0203147] [INSPIRE].

[5] C. Pope, Axial vector anomalies and the index theorem in charged Schwarzschild and Taub-NUT spaces, Nucl. Phys. B 141 (1978) 432 [InSPIRE].

[6] J.P. Gauntlett, N. Kim, J. Park and P. Yi, Monopole dynamics and BPS dyons $N=2$ super Yang-Mills theories, Phys. Rev. D 61 (2000) 125012 [hep-th/9912082] [INSPIRE].

[7] J.R. David and A. Sen, CHL dyons and statistical entropy function from D1 - D5 system, JHEP 11 (2006) 072 [hep-th/0605210] [INSPIRE].

[8] S. Elitzur, A. Forge and E. Rabinovici, Some global aspects of string compactifications, Nucl. Phys. B 359 (1991) 581 [inSPIRE].

[9] G. Mandal, A.M. Sengupta and S.R. Wadia, Classical solutions of two-dimensional string theory, Mod. Phys. Lett. A 6 (1991) 1685 [InSPIRE].

[10] E. Witten, On string theory and black holes, Phys. Rev. D 44 (1991) 314 [InSPIRE].

[11] C.V. Johnson and R.C. Myers, Taub-NUT dyons in heterotic string theory, Phys. Rev. D 50 (1994) 6512 [hep-th/9406069] [InSPIRE].

[12] A. Sen, Twisted black p-brane solutions in string theory, Phys. Lett. B 274 (1992) 34 [hep-th/9108011] [INSPIRE].

[13] S. Hassan and A. Sen, Twisting classical solutions in heterotic string theory, Nucl. Phys. B 375 (1992) 103 [hep-th/9109038] [INSPIRE].

[14] D. Friedan and P. Windey, Supersymmetric Derivation of the Atiyah-Singer Index and the Chiral Anomaly, Nucl. Phys. B 235 (1984) 395 [INSPIRE].

[15] G. Gibbons and C. Pope, $\mathbb{C P}^{2}$ as a gravitational instanton, Commun. Math. Phys. 61 (1978) 239 [INSPIRE].

[16] J.P. Gauntlett and D.A. Lowe, Dyons and $S$ duality in $N=4$ supersymmetric gauge theory, Nucl. Phys. B 472 (1996) 194 [hep-th/9601085] [INSPIRE].

[17] K.-M. Lee, E.J. Weinberg and P. Yi, Electromagnetic duality and SU(3) monopoles, Phys. Lett. B 376 (1996) 97 [hep-th/9601097] [InSPIRE].

[18] R. Dijkgraaf, H.L. Verlinde and E.P. Verlinde, String propagation in a black hole geometry, Nucl. Phys. B 371 (1992) 269 [INSPIRE].

[19] D. Karabali and V. Nair, Quantum Hall effect in higher dimensions, Nucl. Phys. B 641 (2002) 533 [hep-th/0203264] [INSPIRE].

[20] B.P. Dolan, The spectrum of the Dirac operator on coset spaces with homogeneous gauge fields, JHEP 05 (2003) 018 [hep-th/0304037] [INSPIRE].

[21] A. Kirchberg, J. Lange and A. Wipf, Extended supersymmetries and the Dirac operator, Annals Phys. 315 (2005) 467 [hep-th/0401134] [INSPIRE].

[22] D. Israel, A. Pakman and J. Troost, D-branes in $N=2$ Liouville theory and its mirror, Nucl. Phys. B 710 (2005) 529 [hep-th/0405259] [INSPIRE].

[23] T. Eguchi and Y. Sugawara, $\mathrm{SL}(2, \mathbb{R}) / \mathrm{U}(1)$ supercoset and elliptic genera of noncompact Calabi-Yau manifolds, JHEP 05 (2004) 014 [hep-th/0403193] [INSPIRE].

[24] J. Troost, The non-compact elliptic genus: mock or modular, JHEP 06 (2010) 104 [arXiv: 1004.3649] [INSPIRE]. 
[25] T. Eguchi and Y. Sugawara, Non-holomorphic modular forms and $\mathrm{SL}(2, \mathbb{R}) / \mathrm{U}(1)$ Superconformal Field Theory, JHEP 03 (2011) 107 [arXiv: 1012.5721] [INSPIRE].

[26] S.K. Ashok and J. Troost, A twisted non-compact elliptic genus, JHEP 03 (2011) 067 [arXiv:1101.1059] [INSPIRE].

[27] T. Kawai, Y. Yamada and S.-K. Yang, Elliptic genera and $N=2$ superconformal field theory, Nucl. Phys. B 414 (1994) 191 [hep-th/9306096] [INSPIRE].

[28] S. Zwegers, Mock Theta functions, Ph.D. Thesis, Utrecht University, Utrecht Netherlands (2002).

[29] D. Zagier, Ramanujan's mock theta functions and their applications d'après Zwegers and Bringmann-Ono, Séminaire Bourbaki, no. 986 (2007).

[30] R. Akhoury and A. Comtet, Anomalous behavior of the Witten index: exactly soluble models, Nucl. Phys. B 246 (1984) 253 [inSPIRE].

[31] T. Eguchi and K. Hikami, Superconformal Algebras and Mock Theta Functions, J. Phys. A 42 (2009) 304010 [arXiv:0812.1151] [InSPIRE].

[32] M.C. Cheng, J.F. Duncan and J.A. Harvey, Umbral Moonshine, arXiv:1204.2779 [INSPIRE].

[33] S. Alexandrov, J. Manschot and B. Pioline, D3-instantons, mock theta series and twistors, JHEP 04 (2013) 002 [arXiv: 1207.1109] [INSPIRE].

[34] A. Dabholkar, S. Murthy and D. Zagier, Quantum Black Holes, Wall Crossing and Mock Modular Forms, arXiv:1208.4074 [INSPIRE].

[35] L. Carlevaro and D. Israel, Gauge Threshold Corrections for $N=2$ Heterotic Local Models with Flux and Mock Modular Forms, JHEP 03 (2013) 049 [arXiv:1210.5566] [INSPIRE].

[36] D. Bak, C.-k. Lee, K.-M. Lee and P. Yi, Low-energy dynamics for 1/4 BPS dyons, Phys. Rev. D 61 (2000) 025001 [hep-th/9906119] [INSPIRE].

[37] D. Tong, Mirror mirror on the wall: on $2-D$ black holes and Liouville theory, JHEP 04 (2003) 031 [hep-th/0303151] [INSPIRE]. 